\title{
TWO NEW APPROACHES FOR CONSTRUCTION OF THE HIGH ORDER OF ACCURACY DIFFERENCE SCHEMES FOR HYPERBOLIC DIFFERENTIAL EQUATIONS
}

\author{
ALLABEREN ASHYRALYEV AND PAVEL E. SOBOLEVSKII
}

Received 30 March 2004

We consider the abstract Cauchy problem for differential equation of the hyperbolic type $v^{\prime \prime}(t)+A v(t)=f(t)(0 \leq t \leq T), v(0)=v_{0}, v^{\prime}(0)=v_{0}^{\prime}$ in an arbitrary Hilbert space $H$ with the selfadjoint positive definite operator $A$. The high order of accuracy two-step difference schemes generated by an exact difference scheme or by the Taylor decomposition on the three points for the numerical solutions of this problem are presented. The stability estimates for the solutions of these difference schemes are established. In applications, the stability estimates for the solutions of the high order of accuracy difference schemes of the mixed-type boundary value problems for hyperbolic equations are obtained.

\section{The Cauchy problem}

We consider the abstract Cauchy problem for hyperbolic equations

$$
v^{\prime \prime}(t)+A v(t)=f(t) \quad(0 \leq t \leq T), \quad v(0)=v_{0}, \quad v^{\prime}(0)=v_{0}^{\prime}
$$

in a Hilbert space $H$ with the selfadjoint positive definite operator $A$.

A function $v(t)$ is called a solution of the problem (1.1) if the following conditions are satisfied.

(i) $v(t)$ is twice continuously differentiable on the segment $[0, T]$. The derivatives at the endpoints of the segment are understood as the appropriate unilateral derivatives.

(ii) The element $v(t)$ belongs to $D(A)$ for all $t \in[0, T]$, and the function $A v(t)$ is continuous on the segment $[0, T]$.

(iii) $v(t)$ satisfies the equations and the initial conditions (1.1).

It is known (see, e.g., $[7,9]$ ) that various initial boundary value problems for the hyperbolic equations can be reduced to the problem (1.1). A study of discretization, over time only, of the initial value problem also permits one to include general difference schemes in applications, if the differential operator in space variables, $A$, is replaced by the difference operators $A_{h}$ that act in the Hilbert spaces and are uniformly positive definite and selfadjoint in $h$ for $0<h \leq h_{0}$.

In a large cycle of works on high order of accuracy difference schemes for hyperbolic partial differential equations (see, e.g., $[1,10]$ and the references given therein), stability 
was established under the assumption that the magnitudes of the grid steps $\tau$ and $h$ with respect to the time and space variables are connected. In abstract terms this means, in particular, that the condition $\tau\left\|A_{h}\right\| \rightarrow 0$ when $\tau \rightarrow 0$ is satisfied.

Of great interest is the study of absolute stable difference schemes of a high order of accuracy for hyperbolic partial differential equations, in which stability was established without any assumptions with respect to the grid steps $\tau$ and $h$. In $[4,5,12]$ the corresponding simple difference schemes of the first and second order of accuracy for hyperbolic partial differential equations were studied.

In the present paper the two-step difference schemes of a high order of accuracy generated by an exact difference scheme or by the Taylor decomposition on the three points for the numerical solutions of the problem (1.1) are presented. The stability estimates for the solutions of these difference schemes are established. In applications, the stability estimates for the solutions of the high order of accuracy difference schemes of the mixed-type boundary value problems for hyperbolic equations are obtained.

If the function $f(t)$ is not only continuous, but also continuously differentiable on $[0, T], v_{0} \in D(A)$, and $v_{0}^{\prime} \in D\left(A^{1 / 2}\right)$, it is easy to show that the formula

$$
v(t)=c(t) v_{0}+s(t) v_{0}^{\prime}+\int_{0}^{t} s(t-\lambda) f(\lambda) d \lambda
$$

gives a solution of problem (1.1). Here

$$
c(t)=\frac{e^{i t A^{1 / 2}}+e^{-i t A^{1 / 2}}}{2}, \quad s(t)=A^{-1 / 2} \frac{e^{i t A^{1 / 2}}-e^{-i t A^{1 / 2}}}{2 i} .
$$

Actually, obviously (1.1) can be rewritten as the equivalent initial value problem for system of the first-order linear differential equations

$$
\begin{array}{ll}
v^{\prime}(t)+i B v(t)=z(t) \quad & (0 \leq t \leq T), \quad v(0)=v_{0}, \quad v^{\prime}(0)=v_{0}^{\prime}, \\
& z^{\prime}(t)-i B z(t)=f(t),
\end{array}
$$

where $B=A^{1 / 2}$. Integrating these, we at once obtain

$$
\begin{aligned}
& v(t)=e^{-i B t} v_{0}+\int_{0}^{t} e^{-i B(t-s)} z(s) d s, \\
& z(t)=e^{i B t} z_{0}+\int_{0}^{t} e^{i B(t-p)} f(p) d p .
\end{aligned}
$$

That is,

$$
v(t)=e^{-i B t} v_{0}+\int_{0}^{t} e^{-i B(t-s)} e^{i B s} z_{0} d s+\int_{0}^{t} e^{-i B(t-s)} \int_{0}^{s} e^{i B(s-p)} f(p) d p d s,
$$

so, using the condition $v^{\prime}(0)+i B v(0)=z(0)$, we obtain

$$
\begin{aligned}
v(t)= & \left(e^{-i B t}+i B \int_{0}^{t} e^{-i B(t-s)} e^{i B s} d s\right) v_{0}+\int_{0}^{t} e^{-i B(t-s)} e^{i B s} d s v^{\prime}(0) \\
& +\int_{0}^{t} e^{-i B(t-s)} \int_{0}^{s} e^{i B(s-p)} f(p) d p d s .
\end{aligned}
$$


By the interchange of the order of integration, we obtain

$$
\begin{aligned}
v(t)= & \left(e^{-i B t}+\frac{e^{i B t}-e^{-i B t}}{2}\right) v_{0}+B^{-1} \frac{e^{i B t}-e^{-i B t}}{2 i} d s v^{\prime}(0) \\
& +\int_{0}^{t} B^{-1} \frac{e^{i B(t-s)}-e^{-i B(t-s)}}{2 i} f(s) d s .
\end{aligned}
$$

Thus, from that by the definitions of $B, c(t)$, and $s(t)$ we have (1.2).

Theorem 1.1. Suppose that $v_{0} \in D(A), v_{0}^{\prime} \in D\left(A^{1 / 2}\right)$, and $f(t)$ are continuously differentiable on $[0, T]$ function. Then there is a unique solution of the problem (1.1) and the stability inequalities

$$
\begin{gathered}
\max _{0 \leq t \leq T}\|v(t)\|_{H} \leq M\left[\left\|v_{0}\right\|_{H}+\left\|A^{-1 / 2} v_{0}^{\prime}\right\|_{H}+\max _{0 \leq t \leq T}\left\|A^{-1 / 2} f(t)\right\|_{H}\right], \\
\max _{0 \leq t \leq T}\left\|A^{1 / 2} v(t)\right\|_{H} \leq M\left[\left\|A^{1 / 2} v_{0}\right\|_{H}+\left\|v_{0}^{\prime}\right\|_{H}+\max _{0 \leq t \leq T}\|f(t)\|_{H}\right], \\
\max _{0 \leq t \leq T}\left\|\frac{d^{2} v(t)}{d t^{2}}\right\|_{H}+\max _{0 \leq t \leq T}\|A v(t)\|_{H} \leq M\left[\left\|A v_{0}\right\|_{H}+\left\|A^{1 / 2} v_{0}^{\prime}\right\|_{H}+\|f(0)\|_{H}+\int_{0}^{T}\left\|f^{\prime}(t)\right\|_{H} d t\right]
\end{gathered}
$$

hold, where $M$ does not depend on $f(t), t \in[0, T]$, and $v_{0}, v_{0}^{\prime}$.

Proof. Using the formula (1.2) and estimates

$$
\|c(t)\|_{H \rightarrow H} \leq 1, \quad\left\|A^{1 / 2} s(t)\right\|_{H \rightarrow H} \leq 1,
$$

we obtain

$$
\begin{aligned}
\|v(t)\|_{H} \leq & \|c(t)\|_{H \rightarrow H}\left\|v_{0}\right\|_{H}+\left\|A^{1 / 2} s(t)\right\|_{H \rightarrow H}\left\|A^{-1 / 2} v_{0}^{\prime}\right\|_{H} \\
& +\int_{0}^{t}\left\|A^{1 / 2} s(t-\lambda)\right\|_{H \rightarrow H}\left\|A^{-1 / 2} f(\lambda)\right\|_{H} d \lambda \\
\leq & \left\|v_{0}\right\|_{H}+\left\|A^{-1 / 2} v_{0}^{\prime}\right\|_{H}+T \max _{0 \leq t \leq T}\left\|A^{-1 / 2} f(t)\right\|_{H} .
\end{aligned}
$$

Applying $A^{1 / 2}$ to the formula (1.2) and using the estimates (1.10), in a similar manner with (1.10), we obtain

$$
\begin{aligned}
\left\|A^{1 / 2} v(t)\right\|_{H} \leq & \|c(t)\|_{H \rightarrow H}\left\|A^{1 / 2} v_{0}\right\|_{H}+\left\|A^{1 / 2} s(t)\right\|_{H \rightarrow H}\left\|v_{0}^{\prime}\right\|_{H} \\
& +\int_{0}^{t}\left\|A^{1 / 2} s(t-\lambda)\right\|_{H \rightarrow H}\|f(\lambda)\| \|_{H} d \lambda \\
\leq & \left\|A^{1 / 2} v_{0}\right\|_{H}+\left\|v_{0}^{\prime}\right\|_{H}+T \max _{0 \leq t \leq T}\|f(t)\|_{H} .
\end{aligned}
$$

Now, we obtain the estimate for $\|A v(t)\|_{H}$. Applying $A$ to the formula (1.2) and using an integration by parts, we can write

$$
A v(t)=c(t) A v_{0}+A^{1 / 2} s(t) A^{1 / 2} v_{0}^{\prime}+f(T)-c(t) f(0)-\int_{0}^{t} c(t-\lambda) f^{\prime}(\lambda) d \lambda .
$$


Using the last formula and estimates (1.10), we obtain

$$
\begin{aligned}
\|A v(t)\|_{H} \leq & \|c(t)\|_{H \rightarrow H}\left\|A v_{0}\right\|_{H}+\left\|A^{1 / 2} s(t)\right\|_{H \rightarrow H}\left\|A^{1 / 2} v_{0}^{\prime}\right\|_{H} \\
& +\|f(T)\|_{H}+\|c(t)\|_{H \rightarrow H}\|f(0)\|_{H} \\
& +\int_{0}^{t}\|c(t-\lambda)\|_{H \rightarrow H}\left\|f^{\prime}(\lambda)\right\|_{H} d \lambda \\
\leq & \left\|A v_{0}\right\|_{H}+\left\|A^{1 / 2} v_{0}^{\prime}\right\|_{H}+2\|f(0)\|_{H}+2 T \max _{0 \leq t \leq T}\left\|f^{\prime}(t)\right\|_{H} .
\end{aligned}
$$

Then from the last estimate, it follows that

$$
\max _{0 \leq t \leq T}\|A u(t)\|_{H} \leq\left\|A v_{0}\right\|_{H}+\left\|A^{1 / 2} v_{0}^{\prime}\right\|_{H}+2\|f(0)\|_{H}+2 T \max _{0 \leq t \leq T}\left\|f^{\prime}(t)\right\|_{H} .
$$

The estimate for $\max _{0 \leq t \leq T}\left\|d^{2} v(t) / d t^{2}\right\|_{H}$ follows from the last estimate and the triangle inequality.

Remark 1.2. Theorem 1.1 holds in an arbitrary Banach space $E$ under the following assumptions (see, e.g., $[3,8,11]$ and the references given therein):

$$
\|c(t)\|_{E \rightarrow E} \leq M, \quad\left\|A^{1 / 2} s(t)\right\|_{E \rightarrow E} \leq M, \quad 0 \leq t \leq T .
$$

Now, we consider the application of this abstract Theorem 1.1. First, we consider the mixed problem for wave equation

$$
\begin{gathered}
u_{t t}(t, x)-\left(a(x) u_{x}\right)_{x}+u(t, x)=f(t, x), \quad 0 \leq t \leq T, 0 \leq x \leq L, \\
u(0, x)=\varphi(x), \quad u_{t}(0, x)=\psi(x), \quad 0 \leq x \leq L \\
u(t, 0)=u(t, L), \quad u_{x}(t, 0)=u_{x}(t, L), \quad 0 \leq t \leq T .
\end{gathered}
$$

The problem (1.17) has a unique smooth solution $u(t, x)$ for the smooth $a(x)>0(x \in$ $[0, L]), \varphi(x), \psi(x)(x \in[0, L])$, and $f(t, x)(t \in[0, T], x \in[0, L])$ functions. This allows us to reduce the mixed problem (1.17) to the initial value problem (1.1) in Hilbert space $H=L_{2}[0, L]$ with a selfadjoint positive definite operator $A$ defined by (1.17). We give a number of corollaries of the abstract Theorem 1.1 .

THEOREM 1.3. For solutions of the mixed problem (1.17), the stability inequalities

$$
\begin{aligned}
& \max _{0 \leq t \leq T}\|u(t, \cdot)\|_{W_{2}^{1}[0, L]} \\
& \leq M\left[\max _{0 \leq t \leq T}\|f(t, \cdot)\|_{L_{2}[0, L]}+\|\varphi\|_{W_{2}^{1}[0, L]}+\|\psi\|_{L_{2}[0, L]}\right], \\
& \max _{0 \leq t \leq T}\|u(t, \cdot)\|_{W_{2}^{2}[0, L]}+\max _{0 \leq t \leq T}\left\|u_{t t}(t, \cdot)\right\|_{L_{2}[0, L]} \\
& \quad \leq M\left[\max _{0 \leq t \leq T}\left\|f_{t}(t, \cdot)\right\|_{L_{2}[0, L]}+\|f(0, \cdot)\|_{L_{2}[0, L]}+\|\varphi\|_{W_{2}^{2}[0, L]}+\|\psi\|_{W_{2}^{1}[0, L]}\right]
\end{aligned}
$$

hold, where $M$ does not depend on $f(t, x)$ and $\varphi(x), \psi(x)$. 
The proof of this theorem is based on the abstract Theorem 1.1 and the symmetry properties of the space operator generated by the problem (1.17).

Second, let $\Omega \subset \mathbb{R}^{n}$ be the bounded open domain with smooth boundary $S, \bar{\Omega}=\Omega \cup S$. In $[0, T] \times \bar{\Omega}$ we consider the mixed boundary value problem for hyperbolic equations

$$
\begin{gathered}
u_{t t}(t, x)-\sum_{r=1}^{n}\left(a_{r}(x) u_{x_{r}}\right)_{x_{r}}=f(t, x), \quad x=\left(x_{1}, \ldots, x_{n}\right) \in \bar{\Omega}, 0 \leq t \leq T, \\
u(0, x)=\varphi(x), \quad \frac{\partial u(0, x)}{\partial t}=\psi(x), \quad x \in \bar{\Omega}, \\
u(t, x)=0, \quad x \in S, 0 \leq t \leq T,
\end{gathered}
$$

where $a_{r}(x)(x \in \bar{\Omega}), \varphi(x), \psi(x)(x \in \bar{\Omega})$, and $f(t, x)(t \in[0, T], x \in \bar{\Omega})$ are given smooth functions and $a_{r}(x)>0$.

We introduce the Hilbert space $L_{2}(\bar{\Omega})$ which is the space of all the integrable functions defined on $\bar{\Omega}$, equipped with the norm

$$
\|f\|_{L_{2}(\bar{\Omega})}=\left\{\int \cdots \int_{x \in \bar{\Omega}}|f(x)|^{2} d x_{1} \cdots d x_{n}\right\}^{1 / 2} .
$$

The problem (1.19) has a unique smooth solution $u(t, x)$ for the smooth $a_{r}(x)>0$ and $f(t, x)$ functions. This allows us to reduce the mixed problem (1.19) to the initial value problem (1.1) in Hilbert space $H=L_{2}(\bar{\Omega})$ with a selfadjoint positive definite operator $A$ defined by (1.19). We give a number of corollaries of the abstract Theorem 1.1.

Theorem 1.4. For solutions of the mixed problem (1.19), the stability inequalities

$$
\begin{aligned}
& \max _{0 \leq t \leq T}\|u(t, \cdot)\|_{W_{2}^{1}(\bar{\Omega})} \\
& \quad \leq M\left[\max _{0 \leq t \leq T}\|f(t, \cdot)\|_{L_{2}(\bar{\Omega})}+\|\varphi\|_{W_{2}^{1}(\bar{\Omega})}+\|\psi\|_{L_{2}(\bar{\Omega})}\right] \\
&\left.\max _{0 \leq t \leq T}\|u(t, \cdot)\|\right|_{W_{2}^{2}(\bar{\Omega})}+\max _{0 \leq t \leq T}\left\|u_{t t}(t, \cdot)\right\|_{L_{2}(\bar{\Omega})} \\
& \quad \leq M\left[\max _{0 \leq t \leq T}\left\|f_{t}(t, \cdot)\right\|_{L_{2}(\bar{\Omega})}+\|f(0, \cdot)\|_{L_{2}(\bar{\Omega})}+\|\varphi\|_{W_{2}^{2}(\bar{\Omega})}+\|\psi\|_{W_{2}^{1}(\bar{\Omega})}\right]
\end{aligned}
$$

hold, where $M$ does not depend on $f(t, x)$ and $\varphi(x), \psi(x)$.

The proof of this theorem is based on the abstract Theorem 1.1 and the symmetry properties of the space operator generated by the problem (1.19). 
188 High order of accuracy difference schemes for HDE

\section{The high order of accuracy difference schemes generated by an exact difference scheme}

We consider the initial value problem (1.1). On the segment $[0, T]$ we consider a uniform grid

$$
[0, T]_{\tau}=\left\{t_{k}=k \tau, k=0,1, \ldots, N, N \tau=T\right\}
$$

with step $\tau$. Using the formula (1.2), we can establish the following two-step difference scheme for the solution of the initial value problem (1.1)

$$
\begin{gathered}
v\left(t_{k+1}\right)-2 c(\tau) v\left(t_{k}\right)+v\left(t_{k-1}\right) \\
=\int_{t_{k}}^{t_{k+1}} s\left(t_{k+1}-z\right) f(z) d z+\int_{t_{k-1}}^{t_{k}} s\left(z-t_{k-1}\right) f(z) d z, \quad 1 \leq k \leq N-1, \\
v(0)=v_{0}, \\
\tau^{-1}(v(\tau)-v(0))=\tau^{-1}(c(\tau)-I) v(0)+\tau^{-1} s(\tau) v^{\prime}(0)+\tau^{-1} \int_{0}^{\tau} s(\tau-z) f(z) d z
\end{gathered}
$$

or

$$
\begin{gathered}
\frac{1}{\tau^{2}}\left(u_{k+1}-2 u_{k}+u_{k-1}\right)=\frac{2}{\tau^{2}}(c(\tau)-I) u_{k}+f_{k}, \quad f_{k}=\tau^{-1}\left\{f_{1, k+1}+s(\tau) f_{2, k}-c(\tau) f_{1, k}\right\}, \\
f_{1, k}=\tau^{-1} \int_{t_{k-1}}^{t_{k}} s\left(t_{k}-z\right) f(z) d z, \quad f_{2, k}=\tau^{-1} \int_{t_{k-1}}^{t_{k}} c\left(t_{k}-z\right) f(z) d z, \quad 1 \leq k \leq N-1, \\
u_{0}=v_{0}, \quad u_{1}=c(\tau) v(0)+s(\tau) v^{\prime}(0)+\tau f_{1,1} .
\end{gathered}
$$

The latter will be referred to as the exact two-step difference scheme for the initial value problem (1.1).

Suppose the operators $\left(I-e^{2 \tau i B}\right)$ and $\left(I+e^{\tau i B}\right)$ have the bounded inverses $\left(I-e^{2 \tau i B}\right)^{-1}$ and $\left(I+e^{\tau i B}\right)^{-1}$. Then this problem is uniquely solvable, and the following formula holds:

$$
\begin{aligned}
u_{k}= & {\left[e^{-k \tau i B}-\left(I-e^{2 \tau i B}\right)^{-1}\left(e^{-k \tau i B}-e^{k \tau i B}\right)\right] u_{0}+e^{\tau i B}\left(I-e^{2 \tau i B}\right)^{-1}\left(e^{-k \tau i B}-e^{k \tau i B}\right) u_{1} } \\
& +\tau^{2} \sum_{m=1}^{k-1} e^{\tau i B}\left(I-e^{2 \tau i B}\right)^{-1}\left(e^{-(k-m) \tau i B}-e^{(k-m) \tau i B}\right) f_{m}, \quad 2 \leq k \leq N .
\end{aligned}
$$

Actually, (2.3) can be rewritten as the equivalent initial value problem for system of the first-order difference equations:

$$
\begin{gathered}
\tau^{-1}\left(u_{k}-u_{k-1}\right)+\tau^{-1}\left(I-e^{-\tau i B}\right) u_{k-1}=z_{k}, \quad 1 \leq k \leq N, u_{0}, u_{1} \text { are given, } \\
\tau^{-1}\left(z_{k+1}-z_{k}\right)+\tau^{-1}\left(I-e^{\tau i B}\right) z_{k}=f_{k}, \quad 1 \leq k \leq N-1 .
\end{gathered}
$$


From that, the system of recursion formulas follows :

$$
\begin{gathered}
u_{k}=e^{-\tau i B} u_{k-1}+\tau z_{k}, \quad 1 \leq k \leq N, \\
z_{k+1}=e^{\tau i B} z_{k}+\tau f_{k}, \quad 1 \leq k \leq N-1 .
\end{gathered}
$$

Hence

$$
\begin{gathered}
u_{k}=e^{-k \tau i B} u_{0}+\sum_{s=1}^{k} e^{-(k-s) \tau i B} \tau z_{s}, \quad 1 \leq k \leq N, \\
z_{k}=e^{(k-1) i \tau B} z_{1}+\sum_{m=1}^{k-1} e^{(k-1-m) \tau i B} \tau f_{m}, \quad 2 \leq k \leq N .
\end{gathered}
$$

From that it follows that

$$
\begin{gathered}
u_{1}=e^{-\tau i B} u_{0}+\tau z_{1}, \\
u_{k}=e^{-k \tau i B} u_{0}+e^{-(k-1) \tau i B} \tau z_{1}+\sum_{s=2}^{k} e^{-(k-s) \tau i B} \tau\left[e^{(s-1) i \tau B} z_{1}+\sum_{m=1}^{s-1} e^{(s-1-m) \tau i B} \tau f_{m}\right] \\
=e^{-k \tau i B} u_{0}+\left[e^{-(k-1) \tau i B}+\sum_{s=2}^{k} e^{-(k-s) \tau i B} e^{(s-1) i \tau B}\right] \tau z_{1} \\
+\sum_{s=2}^{k} e^{-(k-s) \tau i B} \sum_{m=1}^{s-1} e^{(s-1-m) \tau i B} \tau^{2} f_{m}, \quad 2 \leq k \leq N .
\end{gathered}
$$

Since

$$
\begin{aligned}
e^{-(k-1) \tau i B}+\sum_{s=2}^{k} e^{-(k-s) \tau i B} e^{(s-1) i \tau B} & =\left(I-e^{2 \tau i B}\right)^{-1}\left(e^{-(k-1) \tau i B}-e^{(k+1) \tau i B}\right) \\
& =\left(I-e^{2 \tau i B}\right)^{-1} e^{\tau i B}\left(e^{-k \tau i B}-e^{k \tau i B}\right), \\
\sum_{s=2}^{k} e^{-(k-s) \tau i B} \sum_{m=1}^{s-1} e^{(s-1-m) \tau i B} \tau^{2} f_{m} & =\sum_{m=1}^{k-1} \sum_{s=m+1}^{k} e^{-(k-s) \tau i B} e^{(s-1-m) \tau i B} \tau^{2} f_{m} \\
& =\sum_{m=1}^{k-1}\left(I-e^{2 \tau i B}\right)^{-1} e^{\tau i B}\left(e^{-(k-m) \tau i B}-e^{(k-m) \tau i B}\right) \tau^{2} f_{m},
\end{aligned}
$$

we have that

$$
\begin{aligned}
\mathcal{u}_{k}= & e^{-k \tau i B} u_{0}+\left(I-e^{2 \tau i B}\right)^{-1} e^{\tau i B}\left(e^{-k \tau i B}-e^{k \tau i B}\right) \tau z_{1} \\
& +\sum_{m=1}^{k-1}\left(I-e^{2 \tau i B}\right)^{-1} e^{\tau i B}\left(e^{-(k-m) \tau i B}-e^{(k-m) \tau i B}\right) \tau^{2} f_{m}, \quad 2 \leq k \leq N .
\end{aligned}
$$

Using this formula and the formula $\tau z_{1}=u_{1}-e^{-\tau i B} u_{0}$, we can obtain the formula (2.4).

We investigate the stability of the exact two-step difference scheme (2.3). 
Theorem 2.1. Suppose the operators $\left(I-e^{2 \tau i B}\right)$ and $\left(I+e^{\tau i B}\right)$ have the bounded inverses $\left(I-e^{2 \tau i B}\right)^{-1}$ and $\left(I+e^{\tau i B}\right)^{-1}$. Suppose further that $u_{0} \in D\left(B^{2}\left(I+e^{\tau i B}\right)^{-1}\right), u_{1}-u_{0} \in$ $D\left(B^{2}\left(I-e^{2 \tau i B}\right)^{-1}\right)$, and $f_{k} \in D\left(B\left(I-e^{2 \tau i B}\right)^{-1}\right), 1 \leq k \leq N-1$. Then for the solution of the exact two-step difference scheme (2.3), the following stability inequalities hold:

$$
\begin{aligned}
& \max _{0 \leq k \leq N}\left\|u_{k}\right\|_{H} \\
& \leq M {\left[\max _{1 \leq k \leq N-1}\left\|\tau\left(I-e^{2 \tau i B}\right)^{-1} f_{k}\right\|_{H}+\left\|\left(I-e^{2 \tau i B}\right)^{-1}\left(u_{1}-u_{0}\right)\right\|_{H}\right.} \\
&\left.+\left\|\left(I+e^{\tau i B}\right)^{-1} u_{0}\right\|_{H}\right] \\
& \max _{1 \leq k \leq N}\left\|\tau^{-1}\left(u_{k}-u_{k-1}\right)\right\|_{H}+\max _{1 \leq k \leq N}\left\|\tau^{-1}\left(I-e^{-\tau i B}\right) u_{k-1}\right\|_{H} \\
& \leq M\left[\max _{1 \leq k \leq N-1}\left\|\tau B\left(I-e^{2 \tau i B}\right)^{-1} f_{k}\right\|_{H}+\left\|B\left(I-e^{2 \tau i B}\right)^{-1}\left(u_{1}-u_{0}\right)\right\|_{H}\right. \\
&\left.\quad+\left\|B\left(I+e^{\tau i B}\right)^{-1} u_{0}\right\|_{H}\right], \\
& \max _{1 \leq k \leq N-1}\left\|\tau^{-2}\left(u_{k+1}-2 u_{k}+u_{k-1}\right)\right\|_{H}+\max _{1 \leq k \leq N-1}\left\|\frac{2}{\tau^{2}}(c(\tau)-I) u_{k}\right\|_{H} \\
& \leq M\left[\max _{2 \leq k \leq N-1}\left\|B\left(I-e^{2 \tau i B}\right)^{-1}\left(f_{k}-f_{k-1}\right)\right\|\left\|_{H}+\right\| \tau B\left(I-e^{2 \tau i B}\right)^{-1} f_{1} \|_{H}\right. \\
&\left.\quad\left\|B^{2}\left(I-e^{2 \tau i B}\right)^{-1}\left(u_{1}-u_{0}\right)\right\|_{H}+\left\|B^{2}\left(I+e^{\tau i B}\right)^{-1} u_{0}\right\|_{H}\right]
\end{aligned}
$$

where $M$ does not depend on $\tau, f_{k}, 1 \leq k \leq N-1$, and $u_{0}, u_{1}$.

Proof. Using the formula (2.4) and the estimates

$$
\left\|e^{ \pm k \tau i B}\right\|_{H \rightarrow H} \leq 1, \quad 1 \leq k \leq N,
$$

we obtain

$$
\begin{aligned}
\left\|u_{k}\right\|_{H} \leq & \left\|\left(I+e^{\tau i B}\right) e^{-k \tau i B}-\left(e^{-k \tau i B}-e^{k \tau i B}\right)\right\|_{H \rightarrow H}\left\|\left(I+e^{\tau i B}\right)^{-1} u_{0}\right\|_{H} \\
& +\left\|e^{-k \tau i B}-e^{k \tau i B}\right\|_{H \rightarrow H}\left\|\left(I-e^{2 \tau i B}\right)^{-1}\left(u_{1}-u_{0}\right)\right\|_{H} \\
& +\tau^{2} \sum_{m=1}^{k-1}\left\|e^{\tau i B}\left(e^{-(k-m) \tau i B}-e^{(k-m) \tau i B}\right)\right\|_{H \rightarrow H}\left\|\left(I-e^{2 \tau i B}\right)^{-1} f_{m}\right\|_{H} \\
\leq & M\left[\max _{1 \leq m \leq N-1}\left\|\tau\left(I-e^{2 \tau i B}\right)^{-1} f_{m}\right\|_{H}+\left\|\left(I-e^{2 \tau i B}\right)^{-1}\left(u_{1}-u_{0}\right)\right\|_{H}\right. \\
& \left.+\left\|\left(I+e^{\tau i B}\right)^{-1} u_{0}\right\|_{H}\right]
\end{aligned}
$$

for $k \geq 2$. It obviously holds also for $k=0,1$. The estimate (2.11) is established. Applying $\tau^{-1}\left(I-e^{-\tau i B}\right)$ to the formula (2.4) and using the estimates (2.14) and

$$
\left\|\tau^{-1}\left(I-e^{-\tau i B}\right) B^{-1}\right\|_{H \rightarrow H} \leq 1,
$$


in a similar manner with (2.11), we obtain

$$
\begin{aligned}
&\left\|\tau^{-1}\left(I-e^{-\tau i B}\right) u_{k-1}\right\|_{H} \\
& \leq\left\|\tau^{-1}\left(I-e^{-\tau i B}\right) B^{-1}\right\|_{H \rightarrow H} \\
& \quad \quad\left\{\left\|\left(I+e^{\tau i B}\right) e^{-(k-1) \tau i B}-\left(e^{-(k-1) \tau i B}-e^{(k-1) \tau i B}\right)\right\|_{H \rightarrow H}\left\|B\left(I+e^{\tau i B}\right)^{-1} u_{0}\right\|_{H}\right. \\
& \quad+\left\|e^{-(k-1) \tau i B}-e^{(k-1) \tau i B}\right\|_{H \rightarrow H}\left\|B\left(I-e^{2 \tau i B}\right)^{-1}\left(u_{1}-u_{0}\right)\right\|_{H} \\
&\left.\quad+\tau \sum_{m=1}^{k-2}\left\|e^{\tau i B}\left(e^{-(k-1-m) \tau i B}-e^{(k-1-m) \tau i B}\right)\right\|_{H \rightarrow H}\left\|\tau B\left(I-e^{2 \tau i B}\right)^{-1} f_{m}\right\|_{H}\right\} \\
& \leq M\left[\max _{1 \leq m \leq N-1}\left\|B \tau\left(I-e^{2 \tau i B}\right)^{-1} f_{m}\right\|_{H}+\left\|B\left(I-e^{2 \tau i B}\right)^{-1}\left(u_{1}-u_{0}\right)\right\|_{H}\right. \\
&\left.\quad+\left\|B\left(I+e^{\tau i B}\right)^{-1} u_{0}\right\|_{H}\right]
\end{aligned}
$$

for $k \geq 3$. It obviously holds also for $k=1,2$. So, we have that

$$
\begin{aligned}
\max _{1 \leq k \leq N}\left\|\tau^{-1}\left(I-e^{-\tau i B}\right) u_{k-1}\right\|_{H} \leq M & \max _{1 \leq k \leq N-1}\left\|B \tau\left(I-e^{2 \tau i B}\right)^{-1} f_{k}\right\|_{H} \\
& \left.+\left\|B\left(I-e^{2 \tau i B}\right)^{-1}\left(u_{1}-u_{0}\right)\right\|_{H}+\left\|B\left(I+e^{\tau i B}\right)^{-1} u_{0}\right\|_{H}\right] .
\end{aligned}
$$

In a similar manner, one can show that

$$
\begin{aligned}
\max _{1 \leq k \leq N}\left\|\tau^{-1}\left(u_{k}-u_{k-1}\right)\right\|_{H} \leq M & \max _{1 \leq k \leq N-1}\left\|B \tau\left(I-e^{2 \tau i B}\right)^{-1} f_{k}\right\|_{H} \\
& \left.+\left\|B\left(I-e^{2 \tau i B}\right)^{-1}\left(u_{1}-u_{0}\right)\right\|_{H}+\left\|B\left(I+e^{\tau i B}\right)^{-1} u_{0}\right\|_{H}\right] .
\end{aligned}
$$

The estimate (2.12) is established. Now, we obtain the estimate for $\left\|\left(2 / \tau^{2}\right)(c(\tau)-I) u_{k}\right\|_{H}$. Applying $\left(2 / \tau^{2}\right)(c(\tau)-I)=\left(1 / \tau^{2}\right) e^{-\tau i B}\left(I-e^{\tau i B}\right)^{2}$ to the formula (2.4) and using the Abel formula, we can write the formula

$$
\begin{aligned}
\frac{2}{\tau^{2}}(c(\tau)-I) u_{k}= & \frac{1}{\tau^{2}} e^{-\tau i B}\left(I-e^{\tau i B}\right)^{2}\left[e^{-k \tau i B}-\left(I+e^{\tau i B}\right)^{-1}\left(e^{-k \tau i B}-e^{k \tau i B}\right)\right] u_{0} \\
& +\frac{1}{\tau^{2}}\left(I-e^{\tau i B}\right)^{2}\left(I-e^{2 \tau i B}\right)^{-1}\left(e^{-k \tau i B}-e^{k \tau i B}\right)\left(u_{1}-u_{0}\right) \\
& +\frac{1}{\tau}\left(I-e^{\tau i B}\right) \sum_{m=1}^{k-1} \tau\left(I-e^{\tau i B}\right)\left(I-e^{2 \tau i B}\right)^{-1}\left(e^{-(k-m) \tau i B}-e^{(k-m) \tau i B}\right) f_{m}
\end{aligned}
$$


192

High order of accuracy difference schemes for HDE

$$
\begin{aligned}
= & \frac{1}{\tau^{2}} e^{-\tau i B}\left(I-e^{\tau i B}\right)^{2}\left[e^{-k \tau i B}-\left(I+e^{\tau i B}\right)^{-1}\left(e^{-k \tau i B}-e^{k \tau i B}\right)\right] u_{0} \\
& +\frac{1}{\tau^{2}}\left(I-e^{\tau i B}\right)^{2}\left(I-e^{2 \tau i B}\right)^{-1}\left(e^{-k \tau i B}-e^{k \tau i B}\right)\left(u_{1}-u_{0}\right) \\
& +\frac{1}{\tau}\left(I-e^{\tau i B}\right) \sum_{m=1}^{k-1} \tau\left(I-e^{2 \tau i B}\right)^{-1}\left(e^{-(k-m) \tau i B}+e^{(k+1-m) \tau i B}\right)\left(f_{m}-f_{m-1}\right) \\
& +\frac{1}{\tau}\left(I-e^{\tau i B}\right)\left(e^{-(k-1) \tau i B}+e^{k \tau i B}\right)\left(I-e^{2 \tau i B}\right)^{-1} \tau f_{1} \\
& -\frac{1}{\tau}\left(I-e^{\tau i B}\right)\left(I+e^{\tau i B}\right)\left(I-e^{2 \tau i B}\right)^{-1} \tau f_{k-1} .
\end{aligned}
$$

Using the last formula and estimates (2.14) and (2.16), we obtain

$$
\begin{aligned}
\frac{2}{\tau^{2}} \|(c(\tau) & -I) u_{k} \|_{H} \\
\leq & \left\|\frac{1}{\tau}\left(I-e^{\tau i B}\right) B^{-1}\right\|_{H \rightarrow H}^{2} \\
& \times\left\|e^{-\tau i B}\right\|_{H \rightarrow H}\left\|\left(I+e^{\tau i B}\right) e^{-k \tau i B}-\left(e^{-k \tau i B}-e^{k \tau i B}\right)\right\|_{H \rightarrow H} \\
& \times\left\|B^{2}\left(I+e^{\tau i B}\right)^{-1} u_{0}\right\|_{H}+\left\|\frac{1}{\tau}\left(I-e^{\tau i B}\right) B^{-1}\right\|_{H \rightarrow H}^{2}\left\|e^{-k \tau i B}-e^{k \tau i B}\right\|_{H \rightarrow H} \\
& \times\left\|B^{2}\left(I-e^{2 \tau i B}\right)^{-1}\left(u_{1}-u_{0}\right)\right\|_{H}+\left\|\frac{1}{\tau}\left(I-e^{\tau i B}\right) B^{-1}\right\|_{H \rightarrow H} \\
& \times \sum_{m=1}^{k-1} \tau\left\|\left(e^{-(k-m) \tau i B}+e^{(k+1-m) \tau i B}\right)\right\|\left\|_{H \rightarrow H}\right\| B\left(I-e^{2 \tau i B}\right)^{-1}\left(f_{m}-f_{m-1}\right) \|_{H} \\
& +\left\|\frac{1}{\tau}\left(I-e^{\tau i B}\right) B^{-1}\right\|_{H \rightarrow H}\left\|e^{-(k-1) \tau i B}+e^{k \tau i B}\right\|_{H \rightarrow H}\left\|\tau B\left(I-e^{2 \tau i B}\right)^{-1} f_{1}\right\|_{H} \\
& +\left\|\frac{1}{\tau}\left(I-e^{\tau i B}\right) B^{-1}\right\|_{H \rightarrow H}\left\|I+e^{\tau i B}\right\|_{H \rightarrow H}\left\|\tau B\left(I-e^{2 \tau i B}\right)^{-1} f_{k-1}\right\|_{H} \\
\leq & M\left[\max _{2 \leq m \leq N-1}\left\|B\left(I-e^{2 \tau i B}\right)^{-1}\left(f_{m}-f_{m-1}\right)\right\|\left\|_{H}+\right\| \tau B\left(I-e^{2 \tau i B}\right)^{-1} f_{1} \|_{H}\right. \\
& \left.+\left\|B^{2}\left(I-e^{2 \tau i B}\right)^{-1}\left(u_{1}-u_{0}\right)\right\|\left\|_{H}+\right\| B^{2}\left(I+e^{\tau i B}\right)^{-1} u_{0} \|_{H}\right]
\end{aligned}
$$

for $k \geq 2$. It obviously holds also for $k=1$. So, we have that

$$
\begin{aligned}
\max _{1 \leq k \leq N-1} & \left\|\frac{2}{\tau^{2}}(c(\tau)-I) u_{k}\right\|_{H} \\
\leq M & \max _{2 \leq k \leq N-1}\left\|\tau B\left(I-e^{2 \tau i B}\right)^{-1}\left(f_{k}-f_{k-1}\right)\right\|_{H}+\left\|\tau B\left(I-e^{2 \tau i B}\right)^{-1} f_{1}\right\|_{H} \\
& \left.\quad+\left\|B^{2}\left(I-e^{2 \tau i B}\right)^{-1}\left(u_{1}-u_{0}\right)\right\|_{H}+\left\|B^{2}\left(I+e^{\tau i B}\right)^{-1} u_{0}\right\|_{H}\right] .
\end{aligned}
$$

The estimate for $\max _{1 \leq k \leq N-1}\left\|\tau^{-2}\left(u_{k+1}-2 u_{k}+u_{k-1}\right)\right\|_{H}$ follows from the last estimate and the triangle inequality. 
Note that we have not been able to obtain the following stability inequalities:

$$
\begin{gathered}
\max _{0 \leq k \leq N}\left\|u_{k}\right\|_{H} \leq M\left[\max _{1 \leq k \leq N-1}\left\|B^{-1} f_{k}\right\|_{H}+\left\|B^{-1} \tau^{-1}\left(u_{1}-u_{0}\right)\right\|_{H}+\left\|u_{0}\right\|_{H}\right], \\
\max _{1 \leq k \leq N}\left\|\tau^{-1}\left(u_{k}-u_{k-1}\right)\right\|_{H}+\max _{1 \leq k \leq N}\left\|\tau^{-1}\left(I-e^{-\tau i B}\right) u_{k-1}\right\|_{H} \\
\leq M\left[\max _{1 \leq k \leq N-1}\left\|f_{m}\right\|_{H}+\left\|B u_{0}\right\|_{H}+\left\|\tau^{-1}\left(u_{1}-u_{0}\right)\right\|_{H}\right], \\
\max _{1 \leq k \leq N-1}\left\|\tau^{-2}\left(u_{k+1}-2 u_{k}+u_{k-1}\right)\right\|_{H}+\max _{1 \leq k \leq N-1}\left\|\frac{2}{\tau^{2}}(c(\tau)-I) u_{k}\right\|_{H} \\
\leq M\left[\max _{2 \leq k \leq N-1}\left\|\tau^{-1}\left(f_{k}-f_{k-1}\right)\right\|_{H}+\left\|f_{1}\right\|\left\|_{H}+\right\| B \tau^{-1}\left(u_{1}-u_{0}\right)\left\|_{H}+\right\| B^{2} u_{0} \|_{H}\right]
\end{gathered}
$$

for the solution of the exact two-step difference scheme (2.3). Nevertheless, the following result holds.

Theorem 2.2. Suppose the operators $\left(I-e^{2 \tau i B}\right)$ and $\left(I+e^{\tau i B}\right)$ have the bounded inverses $\left(I-e^{2 \tau i B}\right)^{-1}$ and $\left(I+e^{\tau i B}\right)^{-1}$. Suppose that $u_{0} \in D\left(B^{3}\right), u_{1}-u_{0} \in D\left(B^{2}\right)$, and $f_{k} \in D(B)$, $1 \leq k \leq N-1$. Then for the solution of the exact two-step difference scheme (2.3), the following stability inequalities hold:

$$
\begin{gathered}
\max _{0 \leq k \leq N}\left\|u_{k}\right\|_{H} \leq M\left[\max _{1 \leq k \leq N-1}\left\|f_{k}\right\|_{H}+\left\|\tau^{-1}\left(u_{1}-u_{0}\right)\right\|_{H}+\left\|B u_{0}\right\|_{H}\right] \\
\max _{1 \leq k \leq N}\left\|\tau^{-1}\left(u_{k}-u_{k-1}\right)\right\|_{H}+\max _{1 \leq k \leq N}\left\|\tau^{-1}\left(I-e^{-\tau i B}\right) u_{k-1}\right\|_{H} \\
\leq M\left[\max _{1 \leq k \leq N-1}\left\|B f_{k}\right\|_{H}+\left\|B^{2} u_{0}\right\|_{H}+\left\|B \tau^{-1}\left(u_{1}-u_{0}\right)\right\|_{H}\right] \\
\max _{1 \leq k \leq N-1}\left\|\tau^{-2}\left(u_{k+1}-2 u_{k}+u_{k-1}\right)\right\|_{H}+\max _{1 \leq k \leq N-1}\left\|\frac{2}{\tau^{2}}(c(\tau)-I) u_{k}\right\|_{H} \\
\leq M\left[\max _{2 \leq k \leq N-1}\left\|B \tau^{-1}\left(f_{k}-f_{k-1}\right)\right\|_{H}+\left\|B f_{1}\right\|_{H}+\left\|B^{2} \tau^{-1}\left(u_{1}-u_{0}\right)\right\|_{H}+\left\|B^{3} u_{0}\right\|_{H}\right]
\end{gathered}
$$

where $M$ does not depend on $\tau, f_{k}, 1 \leq k \leq N-1$, and $u_{0}, u_{1}$.

Proof. Using the formula (2.4) and the estimates (2.14), (2.16) and

$$
\left\|\left(I-e^{2 \tau i B}\right)^{-1}\left(e^{-k \tau i B}-e^{k \tau i B}\right)\right\|_{H \rightarrow H} \leq k,
$$


we obtain

$$
\begin{aligned}
\left\|u_{k}\right\|_{H} \leq & \left\|e^{-k \tau i B}\right\|_{H \rightarrow H}\left\|u_{0}\right\|_{H}+\left\|\tau^{-1}\left(I-e^{-\tau i B}\right) B^{-1}\right\|_{H \rightarrow H} \\
& \times\left\|\left(I-e^{2 \tau i B}\right)^{-1}\left(e^{-k \tau i B}-e^{k \tau i B}\right)\right\|_{H \rightarrow H} \tau\left\|B u_{0}\right\|_{H} \\
& +\left\|\left(I-e^{2 \tau i B}\right)^{-1}\left(e^{-k \tau i B}-e^{k \tau i B}\right)\right\|_{H \rightarrow H} \tau\left\|\tau^{-1}\left(u_{1}-u_{0}\right)\right\|_{H} \\
& +\tau^{2} \sum_{m=1}^{k-1}\left\|e^{\tau i B}\right\|_{H \rightarrow H}\left\|\left(I-e^{2 \tau i B}\right)^{-1}\left(e^{-(k-m) \tau i B}-e^{(k-m) \tau i B}\right)\right\|_{H \rightarrow H}\left\|f_{m}\right\|_{H} \\
\leq & M\left[\max _{1 \leq m \leq N-1}\left\|f_{m}\right\|_{H}+\left\|\tau^{-1}\left(u_{1}-u_{0}\right)\right\|_{H}+\left\|B u_{0}\right\|_{H}\right]
\end{aligned}
$$

for $k \geq 2$. It obviously holds also for $k=0,1$. The estimate (2.24) is established. Applying $\tau^{-1}\left(I-e^{-\tau i B}\right)$ to the formula (2.4) and using the estimates (2.14), (2.16), and (2.27) in a similar manner with (2.24), we obtain

$$
\begin{aligned}
& \left\|\tau^{-1}\left(I-e^{-\tau i B}\right) u_{k-1}\right\|_{H} \\
& \leq\left\|\tau^{-1}\left(I-e^{-\tau i B}\right) B^{-1}\right\|_{H \rightarrow H} \\
& \quad \times\left\{\left\|e^{-(k-1) \tau i B}\right\|_{H \rightarrow H}\left\|B u_{0}\right\|_{H}+\left\|\tau^{-1}\left(I-e^{-\tau i B}\right) B^{-1}\right\|_{H \rightarrow H}\right. \\
& \quad \times\left\|\left(I-e^{2 \tau i B}\right)^{-1}\left(e^{-(k-1) \tau i B}-e^{(k-1) \tau i B}\right)\right\|_{H \rightarrow H}\left\|B^{2} u_{0}\right\|_{H} \\
& \quad+\left\|\left(I-e^{2 \tau i B}\right)^{-1}\left(e^{-(k-1) \tau i B}-e^{(k-1) \tau i B}\right)\right\|_{H \rightarrow H} \tau\left\|B \tau^{-1}\left(u_{1}-u_{0}\right)\right\|_{H} \\
& \left.\quad+\tau \sum_{m=1}^{k-2}\left\|e^{\tau i B}\right\|_{H \rightarrow H}\left\|\left(I-e^{2 \tau i B}\right)^{-1}\left(e^{-(k-1-m) \tau i B}-e^{(k-1-m) \tau i B}\right)\right\|_{H \rightarrow H}\left\|\tau B f_{m}\right\|_{H}\right\} \\
& \leq M\left[\max _{1 \leq m \leq N-1}\left\|B f_{m}\right\|_{H}+\left\|B \tau^{-1}\left(u_{1}-u_{0}\right)\right\|_{H}+\left\|B^{2} u_{0}\right\|_{H}\right]
\end{aligned}
$$

for $k \geq 3$. It obviously holds also for $k=1,2$. So, we have that

$$
\max _{1 \leq k \leq N}\left\|\tau^{-1}\left(I-e^{-\tau i B}\right) u_{k-1}\right\|_{H} \leq M\left[\max _{1 \leq k \leq N-1}\left\|B f_{k}\right\|_{H}+\left\|B \tau^{-1}\left(u_{1}-u_{0}\right)\right\|_{H}+\left\|B^{2} u_{0}\right\|_{H}\right] .
$$

In a similar manner, one can show that

$$
\max _{1 \leq k \leq N}\left\|\tau^{-1}\left(u_{k}-u_{k-1}\right)\right\|_{H} \leq M\left[\max _{1 \leq k \leq N-1}\left\|B f_{k}\right\|_{H}+\left\|B \tau^{-1}\left(u_{1}-u_{0}\right)\right\|_{H}+\left\|B^{2} u_{0}\right\|_{H}\right] .
$$


The estimate (2.25) is established. Now, we obtain the estimate for $\left\|\left(2 / \tau^{2}\right)(c(\tau)-I) u_{k}\right\|_{H}$. Using the formula (2.20) and the estimates (2.14), (2.16), and (2.27), we obtain

$$
\begin{aligned}
& \frac{2}{\tau^{2}}\left\|(c(\tau)-I) u_{k}\right\|_{H} \\
& \leq\left\|\frac{1}{\tau}\left(I-e^{\tau i B}\right) B^{-1}\right\|_{H \rightarrow H}^{2}\left\|e^{-\tau i B}\right\|_{H \rightarrow H} \\
& \times\left[\left\|e^{-k \tau i B}\right\|_{H \rightarrow H} \times\left\|B^{2} u_{0}\right\|_{H}+\left\|\frac{1}{\tau}\left(I-e^{\tau i B}\right) B^{-1}\right\|_{H \rightarrow H}\right. \\
& \left.\times\left\|\left(I-e^{2 \tau i B}\right)^{-1}\left(e^{-k \tau i B}-e^{k \tau i B}\right)\right\|_{H \rightarrow H}\left\|B^{3} u_{0}\right\|_{H}\right] \\
& +\left\|\frac{1}{\tau}\left(I-e^{\tau i B}\right) B^{-1}\right\|_{H \rightarrow H}^{2}\left\|\left(I-e^{2 \tau i B}\right)^{-1}\left(e^{-k \tau i B}-e^{k \tau i B}\right)\right\|_{H \rightarrow H}\left\|B^{2}\left(u_{1}-u_{0}\right)\right\|_{H} \\
& +\left\|\frac{1}{\tau}\left(I-e^{\tau i B}\right) B^{-1}\right\|_{H \rightarrow H} \sum_{m=1}^{k-1} \tau\left\|\left(I-e^{2 \tau i B}\right)^{-1}\left(e^{-(k-m) \tau i B}-e^{(k-m) \tau i B}\right)\right\|_{H \rightarrow H} \\
& \times\left\|B\left(f_{m}-f_{m-1}\right)\right\|_{H}+\sum_{m=1}^{k-1}\left\|\left(I-e^{\tau i B}\right)\left(I-e^{2 \tau i B}\right)^{-1}\left(e^{(k-m) \tau i B}+e^{(k+1-m) \tau i B}\right)\right\|_{H \rightarrow H} \\
& \times\left\|\left(f_{m}-f_{m-1}\right)\right\|_{H}+\left\|\frac{1}{\tau}\left(I-e^{\tau i B}\right) B^{-1}\right\|_{H \rightarrow H} \\
& \times\left\|\left(I-e^{2 \tau i B}\right)^{-1}\left[e^{-(k-1) \tau i B}-e^{(k-1) \tau i B}\right]\right\|_{H \rightarrow H} \\
& \times\left\|\tau B f_{1}\right\|_{H}+\left\|\left(I-e^{\tau i B}\right)\left(I-e^{2 \tau i B}\right)^{-1}\left[e^{(k-1) \tau i B}+e^{k \tau i B}\right]\right\|_{H \rightarrow H}\left\|f_{1}\right\|_{H} \\
& +\left\|\left(I-e^{\tau i B}\right)\left(I-e^{2 \tau i B}\right)^{-1}\left[I+e^{\tau i B}\right]\right\|_{H \rightarrow H}\left\|f_{k-1}\right\|_{H} \\
& \leq M\left[\max _{2 \leq m \leq N-1}\left\|B \tau^{-1}\left(f_{m}-f_{m-1}\right)\right\|_{H}+\max _{2 \leq m \leq N-1}\left\|\tau^{-1}\left(f_{m}-f_{m-1}\right)\right\|_{H}\right. \\
& \left.+\left\|B f_{1}\right\|_{H}+\left\|f_{1}\right\|_{H}+\left\|B^{2} \tau^{-1}\left(u_{1}-u_{0}\right)\right\|_{H}+\left\|B^{3} u_{0}\right\|_{H}+\left\|B^{2} u_{0}\right\|_{H}\right] \\
& \leq M_{1}\left[\max _{2 \leq m \leq N-1}\left\|B \tau^{-1}\left(f_{m}-f_{m-1}\right)\right\|_{H}+\left\|B f_{1}\right\|_{H}+\left\|B^{2} \tau^{-1}\left(u_{1}-u_{0}\right)\right\|_{H}+\left\|B^{3} u_{0}\right\|_{H}\right]
\end{aligned}
$$

for $k \geq 2$. It obviously holds also for $k=1$. So, we have that

$$
\begin{aligned}
& \max _{1 \leq k \leq N-1}\left\|\frac{2}{\tau^{2}}(c(\tau)-I) u_{k}\right\|_{H} \\
& \quad \leq M_{1}\left[\max _{2 \leq k \leq N-1}\left\|B \tau^{-1}\left(f_{k}-f_{k-1}\right)\right\|_{H}+\left\|B f_{1}\right\|_{H}+\left\|B^{2} \tau^{-1}\left(u_{1}-u_{0}\right)\right\|_{H}+\left\|B^{3} u_{0}\right\|_{H}\right] .
\end{aligned}
$$

The estimate for $\max _{1 \leq k \leq N-1}\left\|\tau^{-2}\left(u_{k+1}-2 u_{k}+u_{k-1}\right)\right\|_{H}$ follows from the last estimate and the triangle inequality. 
Remark 2.3. Theorem 2.1 actually holds in an arbitrary Banach space $E$ under the following assumptions:

$$
\left\|e^{ \pm k \tau i B}\right\|_{E \rightarrow E} \leq M, \quad 1 \leq k \leq N,
$$

where $M$ does not depend on $\tau$.

Remark 2.4. This approach permits us to construct the high order of accuracy two-step uniform difference schemes for differential equations of the hyperbolic type with an arbitrary parameter $\varepsilon$ on the highest derivative. In $[2,3]$ the stability estimates of the solutions of the high order of accuracy difference schemes for hyperbolic equations with an arbitrary $\varepsilon$ parameter on the highest derivative were obtained.

Now, we will consider the applications of the exact difference scheme (2.3). From (2.3) it is clear that for the approximate solutions of the problem (1.1), it is necessary to approximate the expressions

$$
\begin{gathered}
s(\tau), \quad c(\tau), \\
\int_{t_{k-1}}^{t_{k}} s\left(t_{k}-z\right) f(z) d z, \quad \int_{t_{k-1}}^{t_{k}} c\left(t_{k}-z\right) f(z) d z .
\end{gathered}
$$

We remark that in constructing difference schemes, it is important to know how to construct $f_{k}^{j, l}$ and $f_{1,1}^{j, l}$ such that

$$
\begin{gathered}
\tau^{-2} \int_{t_{k}}^{t_{k+1}} s\left(t_{k+1}-z\right) f(z) d z-c(\tau) \tau^{-2} \int_{t_{k-1}}^{t_{k}} s\left(t_{k}-z\right) f(z) d z \\
+s(\tau) \tau^{-2} \int_{t_{k-1}}^{t_{k}} c\left(t_{k}-z\right) f(z) d z-f_{k}^{j, l}=o\left(\tau^{j+l}\right), \\
\tau^{-1} \int_{0}^{\tau} s(\tau-z) f(z) d z-f_{1,1}^{j, l}=o\left(\tau^{j+l}\right),
\end{gathered}
$$

and the formulas of $f_{k}^{j, l}$ and $f_{1,1}^{j, l}$ are sufficiently simple. The choice of $f_{k}^{j, l}$ and $f_{1,1}^{j, l}$ is not unique. Using Taylor's formula and integration by parts, we obtain the representation

$$
\begin{aligned}
& \tau^{-2} \int_{t_{k-1}}^{t_{k}} s\left(t_{k}-z\right) f(z) d z \\
& \quad=\sum_{m=0}^{j+l-1} \beta_{m} f^{(m)}\left(t_{k-1}\right)+\tau^{-2} \int_{t_{k-1}}^{t_{k}} s\left(t_{k}-z\right) \int_{t_{k-1}}^{s} \frac{(s-z)^{j+l-1}}{(j+l-1) !} f^{(j+l)}(z) d z d s
\end{aligned}
$$

in which

$$
\begin{aligned}
& \beta_{m}=-A^{-1} \frac{\tau^{m-2}}{m !}+A^{-1} \beta_{m-2}, \quad 2 \leq m \leq j+l-1, \\
& \beta_{0}=\tau^{-2} A^{-1}(c(\tau)-I), \quad \beta_{1}=\tau^{-2} A^{-1}(s(\tau)-\tau I) .
\end{aligned}
$$


In a similar manner, one can obtain that

$$
\begin{aligned}
& \tau^{-2} \int_{t_{k-1}}^{t_{k}} c\left(t_{k}-z\right) f(z) d z \\
& \quad=\sum_{m=0}^{j+l-1} \gamma_{m} f^{(m)}\left(t_{k-1}\right)+\tau^{-2} \int_{t_{k-1}}^{t_{k}} c\left(t_{k}-z\right) \int_{t_{k-1}}^{s} \frac{(s-z)^{j+l-1}}{(j+l-1) !} f^{(j+l)}(z) d z d s,
\end{aligned}
$$

in which

$$
\gamma_{m}=\beta_{m-1}, \quad 1 \leq m \leq j+l-1, \quad \gamma_{0}=\tau^{-2} s(\tau) .
$$

Using the definitions of $s(\tau)$ and $c(\tau)$ and Pade fractions for the function $e^{-z}$ (see [1]), we can write

$$
\begin{aligned}
& c(\tau)=\frac{R_{j, l+1}(i \tau B)+R_{j, l+1}(-i \tau B)}{2}+o\left(\tau^{j+l+2}\right), \\
& s(\tau)=B^{-1} \frac{R_{j, l+1}(i \tau B)-R_{j, l+1}(-i \tau B)}{2 i}+o\left(\tau^{j+l+2}\right) .
\end{aligned}
$$

Now, using the formulas (2.36), (2.37), (2.39), and (2.41), $f_{k}^{j, l}$ and $f_{1,1}^{j, l}$ can be defined by the following formulas:

$$
\begin{gathered}
f_{k}^{j, l}=\sum_{m=0}^{j+l-1} B_{m} f^{(m)}\left(t_{k}\right)+B^{-1} \frac{R_{j, l+1}(i \tau B)-R_{j, l+1}(-i \tau B)}{2 i} \sum_{m=0}^{j+l-1} \widetilde{B}_{m} f^{(m)}\left(t_{k-1}\right) \\
-\frac{R_{j, l+1}(i \tau B)+R_{j, l+1}(-i \tau B)}{2} \sum_{m=0}^{j+l-1} B_{m} f^{(m)}\left(t_{k-1}\right), \\
f_{1,1}^{j, l}=\sum_{m=0}^{j+l-2} \widetilde{B}_{m} f^{(m)}(0), \quad f_{0}^{j, l}=\tau f_{1,1}^{j, l},
\end{gathered}
$$

where

$$
\begin{aligned}
B_{m} & =-A^{-1} \frac{\tau^{m-2}}{m !}+A^{-1} B_{m-2}, \quad 2 \leq m \leq j+l-1, \\
B_{0} & =\tau^{-2} A^{-1}\left(\frac{R_{j, l+1}(i \tau B)+R_{j, l+1}(-i \tau B)}{2}-I\right), \\
B_{1} & =\tau^{-2} A^{-1}\left(B^{-1} \frac{R_{j, l+1}(i \tau B)-R_{j, l+1}(-i \tau B)}{2 i}-\tau I\right), \\
\widetilde{B}_{m} & =B_{m-1}, \quad 1 \leq m \leq j+l-1, \\
\widetilde{B}_{0} & =\tau^{-2} B^{-1} \frac{R_{j, l+1}(i \tau B)-R_{j, l+1}(-i \tau B)}{2 i} .
\end{aligned}
$$


Now, using the formulas (2.41), (2.42), and (2.37), we obtain the difference schemes $(j+l)$ th order of accuracy

$$
\begin{gathered}
\tau^{-2}\left(u_{k+1}-2 u_{k}+u_{k-1}\right)+\tilde{A} u_{k}=f_{k}^{j, l}, \quad 1 \leq k \leq N-1, \quad u_{0}=v_{0}, \\
\tilde{A}=2 \tau^{-2}\left(I-\frac{R_{j, l+1}(i \tau B)+R_{j, l+1}(-i \tau B)}{2}\right) \\
\tau^{-1}\left(u_{1}-u_{0}\right)=\tau^{-1}\left(\frac{R_{j, l}(i \tau B)+R_{j, l}(-i \tau B)}{2}-I\right) v_{0} \\
+\tau^{-1} B^{-1} \frac{R_{j, l}(i \tau B)-R_{j, l}(-i \tau B)}{2 i} v^{\prime}(0)+f_{0}^{j, l}
\end{gathered}
$$

for the approximate solution of the initial value problem (1.1).

Note that the difference schemes (2.44) for $j=l, j=l-1$, and $j=l+1$ include difference schemes of arbitrary high order of approximation. Moreover, the corresponding functions $\left|R_{j, l+1}(z)\right|$ tend to 0 as $z \rightarrow \infty$ for $j=l-1, l$ and $\left|R_{j, l+1}(z)\right|=1$ for $j=l+1$. Such difference schemes are simplest, in the sense that the degrees of the denominators of the corresponding Pade approximants of the function $\exp \{-z\}$ are minimal for a fixed order of approximation of the difference schemes.

Suppose the operators $\left(I-R_{j, l}^{2}(i \tau B)\right)$ and $\left(I+R_{j, l}(i \tau B)\right)$ have the bounded inverses $\left(I-R_{j, l}^{2}(i \tau B)\right)^{-1}$ and $\left(I+R_{j, l}(i \tau B)\right)^{-1}$. It is clear that this problem is uniquely solvable, and the following formula holds:

$$
\begin{aligned}
& u_{k}= {\left[R_{j, l}^{k}(-i \tau B)-\left(I-R_{j, l}^{2}(i \tau B)\right)^{-1}\left(R_{j, l}^{k}(-i \tau B)-R_{j, l}^{k}(i \tau B)\right)\right] u_{0} } \\
&+R_{j, l}(i \tau B)\left(I-R_{j, l}^{2}(i \tau B)\right)^{-1}\left(R_{j, l}^{k}(-i \tau B)-R_{j, l}^{k}(i \tau B)\right) u_{1} \\
&+\tau^{2} \sum_{m=1}^{k-1} R_{j, l}(i \tau B)\left(I-R_{j, l}^{2}(i \tau B)\right)^{-1}\left(R_{j, l}^{k-m}(-i \tau B)-R_{j, l}^{k-m}(i \tau B)\right) f_{m}^{j, l}, \quad 2 \leq k \leq N, \\
& u_{1}=\frac{R_{j, l}(i \tau B)+R_{j, l}(-i \tau B)}{2} v_{0}+B^{-1} \frac{R_{j, l}(i \tau B)-R_{j, l}(-i \tau B)}{2 i} v^{\prime}(0)+\tau f_{0}^{j, l}, \\
& u_{0}=v_{0} .
\end{aligned}
$$

We investigate the stability of the exact two-step difference scheme (2.45).

Theorem 2.5. Suppose the operators $\left(I-R_{j, l}^{2}(i \tau B)\right)$ and $\left(I+R_{j, l}(i \tau B)\right)$ have the bounded inverses $\left(I-R_{j, l}^{2}(i \tau B)\right)^{-1}$ and $\left(I+R_{j, l}(i \tau B)\right)^{-1}$. Suppose that $u_{0} \in D\left(B^{2}\right), u_{1}-u_{0} \in D(B)$. Then for the solution of the two-step difference scheme (2.45) for $j=l$ and $j=l-1$, the 
following stability inequalities hold:

$$
\begin{aligned}
& \max _{0 \leq k \leq N}\left\|u_{k}\right\|_{H} \leq M\left[\max _{1 \leq k \leq N-1}\left\|B^{-1} f_{k}^{j, l}\right\|_{H}+\left\|B^{-1} \tau^{-1}\left(u_{1}-u_{0}\right)\right\|_{H}+\left\|u_{0}\right\|_{H}\right], \\
& \max _{1 \leq k \leq N}\left\|\tau^{-1}\left(u_{k}-u_{k-1}\right)\right\|_{H}+\max _{1 \leq k \leq N}\left\|\tau^{-1}\left(I-e^{-\tau i B}\right) u_{k-1}\right\|_{H} \\
& \quad \leq M\left[\max _{1 \leq k \leq N-1}\left\|f_{k}^{j, l}\right\|_{H}+\left\|\tau^{-1}\left(u_{1}-u_{0}\right)\right\|_{H}+\left\|B u_{0}\right\|_{H}\right], \\
& \max _{1 \leq k \leq N-1}\left\|\tau^{-2}\left(u_{k+1}-2 u_{k}+u_{k-1}\right)\right\|_{H}+\max _{1 \leq k \leq N-1}\left\|\tilde{A} u_{k}\right\|_{H} \\
& \leq M\left[\max _{2 \leq k \leq N-1}\left\|\tau^{-1}\left(f_{k}^{j, l}-f_{k-1}^{j, l}\right)\right\|_{H}+\left\|f_{1}^{j, l}\right\|_{H}+\left\|B \tau^{-1}\left(u_{1}-u_{0}\right)\right\|_{H}+\left\|B^{2} u_{0}\right\|_{H}\right],
\end{aligned}
$$

where $M$ does not depend on $\tau, f_{k}^{j, l}, 1 \leq k \leq N-1$, and $u_{0}, u_{1}$.

The proof of Theorem 2.5 follows the scheme of the proof of Theorem 2.1, and relies on the formula (2.45) and on the estimates

$$
\begin{gathered}
\left\|R_{j, l+1}( \pm i \tau B)\right\|_{H \rightarrow H} \leq 1, \\
\left\|\tau B\left(I-R_{j, l+1}^{2}(i \tau B)\right)^{-1} R_{j, l+1}(i \tau B)\right\|_{H \rightarrow H} \leq M .
\end{gathered}
$$

Note that the estimate (2.48) is not satisfied for $j=l+1$. Therefore we have not been able to obtain the same stability inequalities for the solution of the exact two-step difference scheme (2.45) for $j=l+1$. Nevertheless, the following result holds.

Theorem 2.6. Suppose the operators $\left(I-R_{j, l}^{2}(i \tau B)\right)$ and $\left(I+R_{j, l}(i \tau B)\right)$ have the bounded inverses $\left(I-R_{j, l}^{2}(i \tau B)\right)^{-1}$ and $\left(I+R_{j, l}(i \tau B)\right)^{-1}$. Suppose that $u_{0} \in D\left(B^{3}\right), u_{1}-u_{0} \in D\left(B^{2}\right)$, and $f_{k}^{j, l} \in D(B), 1 \leq k \leq N-1$. Then for the solution of the two-step difference scheme (2.45) for $j=l+1$, the following stability inequalities hold:

$$
\begin{gathered}
\max _{0 \leq k \leq N}\left\|u_{k}\right\|_{H} \leq M\left[\max _{1 \leq k \leq N-1}\left\|f_{k}^{j, l}\right\|_{H}+\left\|\tau^{-1}\left(u_{1}-u_{0}\right)\right\|_{H}+\left\|B u_{0}\right\|_{H}\right], \\
\max _{1 \leq k \leq N}\left\|\tau^{-1}\left(u_{k}-u_{k-1}\right)\right\|_{H}+\max _{1 \leq k \leq N}\left\|\tau^{-1}\left(I-e^{-\tau i B}\right) u_{k-1}\right\|_{H} \\
\leq M\left[\max _{1 \leq k \leq N-1}\left\|B f_{k}^{j, l}\right\|_{H}+\left\|B \tau^{-1}\left(u_{1}-u_{0}\right)\right\|_{H}+\left\|B^{2} u_{0}\right\|_{H}\right], \\
\max _{1 \leq k \leq N-1}\left\|\tau^{-2}\left(u_{k+1}-2 u_{k}+u_{k-1}\right)\right\|_{H}+\max _{1 \leq k \leq N-1}\left\|\tilde{A} u_{k}\right\|_{H} \\
\leq M\left[\max _{2 \leq k \leq N-1}\left\|\tau^{-1} B\left(f_{k}^{j, l}-f_{k-1}^{j, l}\right)\right\|_{H}+\left\|B f_{1}^{j, l}\right\|_{H}+\left\|B^{2} \tau^{-1}\left(u_{1}-u_{0}\right)\right\|_{H}+\left\|B^{3} u_{0}\right\|_{H}\right],
\end{gathered}
$$

where $M$ does not depend on $\tau, f_{k}^{j, l}, 1 \leq k \leq N-1$, and $u_{0}, u_{1}$. 
The proof of Theorem 2.6 follows the scheme of the proof of Theorem 2.2, and relies on the formula (2.45), the estimates (2.48), and

$$
\left\|\left(I-R_{j, l+1}(2 i \tau B)\right)^{-1}\left(R_{j, l+1}^{k}(-i \tau B)-R_{j, l+1}^{k}(i \tau B)\right)\right\|_{H \rightarrow H} \leq k .
$$

Remark 2.7. Theorem 2.6 actually holds in an arbitrary Banach space $E$ under the following assumptions:

$$
\left\|R_{j, l+1}^{k}( \pm i \tau B)\right\|_{E \rightarrow E} \leq M, \quad 1 \leq k \leq N
$$

where $M$ does not depend on $\tau$.

Now, the abstract Theorems 2.5 and 2.6 are applied in the investigation of difference schemes of higher order of accuracy with respect to one variable for approximate solutions of the mixed boundary value problem (1.19). The discretization of problem (1.19) is carried out in two steps. In the first step we define the grid sets

$$
\begin{aligned}
\widetilde{\Omega}_{h}=\left\{x=x_{m}\right. & =\left(h_{1} m_{1}, \ldots, h_{n} m_{n}\right), m=\left(m_{1}, \ldots, m_{n}\right), \\
0 \leq m_{r} & \left.\leq N_{r}, h_{r} N_{r}=L, r=1, \ldots, n\right\}, \\
\Omega_{h} & =\widetilde{\Omega}_{h} \cap \Omega, \quad S_{h}=\widetilde{\Omega}_{h} \cap S .
\end{aligned}
$$

We introduce the Banach space $L_{2}\left(\widetilde{\Omega}_{h}\right)$ of the grid functions $\varphi^{h}(x)=\left\{\varphi\left(h_{1} m_{1}, \ldots, h_{n} m_{n}\right)\right\}$ defined on $\widetilde{\Omega}_{h}$, equipped with the norm

$$
\left\|\varphi^{h}\right\|_{L_{2}\left(\widetilde{\Omega}_{h}\right)}=\left(\sum_{x \in \bar{\Omega}_{h}}\left|\varphi^{h}(x)\right|^{2} h_{1} \cdots h_{n}\right)^{1 / 2} .
$$

To the differential operator $A^{x}$ generated by the problem (1.19), we assign the difference operator $A_{h}^{x}$ by the formula

$$
A_{h}^{x} u_{x}^{h}=-\sum_{r=1}^{n}\left(a_{r}(x) u_{\bar{x}_{r}}^{h}\right)_{x_{r}, j_{r}}
$$

acting in the space of grid functions $u^{h}(x)$, and satisfying the conditions $u^{h}(x)=0$ for all $x \in S_{h}$. It is known that $A_{h}^{x}$ is a selfadjoint positive definite operator in $L_{2}\left(\widetilde{\Omega}_{h}\right)$. With the help of $A_{h}^{x}$, we arrive at the initial value problem

$$
\begin{gathered}
\frac{d^{2} v^{h}(t, x)}{d t^{2}}+A_{h}^{x} v^{h}(t, x)=f^{h}(t, x), \quad 0 \leq t \leq T, x \in \widetilde{\Omega}_{h}, \\
v^{h}(0, x)=\varphi^{h}(x), \quad \frac{d v^{h}(0, x)}{d t}=\psi^{h}(x), \quad x \in \widetilde{\Omega}_{h}
\end{gathered}
$$

for an infinite system of ordinary differential equations. 
In the second step we replace problem (2.56) by the difference schemes (2.44):

$$
\begin{gathered}
\tau^{-2}\left(u_{k+1}^{h}(x)-2 u_{k}^{h}(x)+u_{k-1}^{h}(x)\right)+\widetilde{A_{h}^{x}} u_{k}^{h}(x)=f_{k}^{h}(x), \\
t_{k}=k \tau, \quad 1 \leq k \leq N-1, \quad x \in \widetilde{\Omega}_{h}, \quad u_{0}^{h}(x)=\varphi^{h}(x), \\
\tau^{-1}\left(u_{1}^{h}(x)-u_{0}^{h}(x)\right)=\tau^{-1}\left(\frac{R_{j, l}\left(i \tau B_{h}^{x}\right)+R_{j, l}\left(-i \tau B_{h}^{x}\right)}{2}-I\right) \varphi^{h}(x) \\
\quad+\tau^{-1}\left(B_{h}^{x}\right)^{-1} \frac{R_{j, l}\left(i \tau B_{h}^{x}\right)-R_{j, l}\left(-i \tau B_{h}^{x}\right)}{2 i} \psi^{h}(x)+\tau f_{0}^{h}(x), \quad x \in \widetilde{\Omega}_{h}, \\
\widetilde{A}_{h}^{x}=2 \tau^{-2}\left(I-\frac{R_{j, l+1}\left(i \tau B_{h}^{x}\right)+R_{j, l+1}\left(-i \tau B_{h}^{x}\right)}{2}\right), \quad\left(B_{h}^{x}\right)^{2}=A_{h}^{x}, \\
f_{k}^{h}(x)=\sum_{m=0}^{j+l-1} B_{m} f^{(m)}\left(t_{k}, x\right)+B^{-1} \frac{R_{j, l+1}\left(i \tau B_{h}^{x}\right)-R_{j, l+1}\left(-i \tau B_{h}^{x}\right)}{2 i} \\
\times \sum_{m=0}^{j+l-1} \widetilde{B}_{m} f^{(m)}\left(t_{k-1}, x\right)-\frac{R_{j, l+1}\left(i \tau B_{h}^{x}\right)+R_{j, l+1}\left(-i \tau B_{h}^{x}\right)}{2} \sum_{m=0}^{j+l-1} B_{m} f^{(m)}\left(t_{k-1}, x\right), \\
f_{0}^{h}(x)=\tau \sum_{m=0}^{j+l-2} \widetilde{B}_{m} f^{(m)}(0, x),
\end{gathered}
$$

where

$$
\begin{gathered}
B_{m}=-\left(A_{h}^{x}\right)^{-1} \frac{\tau^{m-2}}{m !}+\left(A_{h}^{x}\right)^{-1} B_{m-2}, \quad 2 \leq m \leq j+l-1, \\
B_{0}=\tau^{-2}\left(A_{h}^{x}\right)^{-1}\left(\frac{R_{j, l+1}\left(i \tau B_{h}^{x}\right)+R_{j, l+1}\left(-i \tau B_{h}^{x}\right)}{2}-I\right), \\
B_{1}=\tau^{-2}\left(A_{h}^{x}\right)^{-1}\left(\left(B_{h}^{x}\right)^{-1} \frac{R_{j, l+1}\left(i \tau B_{h}^{x}\right)-R_{j, l+1}\left(-i \tau B_{h}^{x}\right)}{2 i}-\tau I\right), \\
\widetilde{B}_{m}=B_{m-1}, \quad 1 \leq m \leq j+l-1, \\
\widetilde{B}_{0}=\tau^{-2}\left(B_{h}^{x}\right)^{-1} \frac{R_{j, l+1}\left(i \tau B_{h}^{x}\right)-R_{j, l+1}\left(-i \tau B_{h}^{x}\right)}{2 i} .
\end{gathered}
$$

THeOREM 2.8. Let $\tau$ and $|h|$ be sufficiently small numbers. Then the solutions of the difference schemes (2.56) for $j=l$ and $j=l-1$ satisfy the following stability estimates:

$$
\begin{aligned}
& \max _{0 \leq k \leq N}\left\|u_{k}^{h}\right\|_{L_{2}\left(\tilde{\Omega}_{h}\right)} \\
& \quad \leq M_{1}\left[\max _{0 \leq k \leq N-1}\left\|\left(B_{h}^{x}\right)^{-1} f_{k}^{h}\right\|_{L_{2}\left(\tilde{\Omega}_{h}\right)}+\left\|\left(B_{h}^{x}\right)^{-1} \psi^{h}\right\|_{L_{2}\left(\tilde{\Omega}_{h}\right)}+\left\|\varphi^{h}\right\|_{L_{2}\left(\tilde{\Omega}_{h}\right)}\right],
\end{aligned}
$$




$$
\begin{aligned}
& \max _{1 \leq k \leq N}\left\|\tau^{-1}\left(u_{k}^{h}-u_{k-1}^{h}\right)\right\|_{L_{2}\left(\tilde{\Omega}_{h}\right)} \\
& \leq M_{1}\left[\max _{0 \leq k \leq N-1}\left\|f_{k}^{h}\right\|_{L_{2}\left(\tilde{\Omega}_{h}\right)}+\left\|\psi^{h}\right\|_{L_{2}\left(\widetilde{\Omega}_{h}\right)}+\sum_{r=1}^{n}\left\|\varphi_{x_{r}, \bar{m}_{r}}^{h}\right\|_{L_{2}\left(\tilde{\Omega}_{h}\right)}\right], \\
& \max _{1 \leq k \leq N-1}\left\|\tau^{-2}\left(u_{k+1}^{h}-2 u_{k}^{h}+u_{k-1}^{h}\right)\right\|_{L_{2}\left(\tilde{\Omega}_{h}\right)} \\
& \leq M_{1}\left[\max _{1 \leq k \leq N-1}\left\|\tau^{-1}\left(f_{k}^{h}-f_{k-1}^{h}\right)\right\|_{L_{2}\left(\tilde{\Omega}_{h}\right)}+\left\|f_{0}^{h}\right\|_{L_{2}\left(\tilde{\Omega}_{h}\right)}+\sum_{r=1}^{n} \tau^{-1}\left\|\psi_{x_{r}, \bar{m}_{r}}^{h}\right\|_{L_{2}\left(\tilde{\Omega}_{h}\right)}\right. \\
&\left.\quad+\sum_{r=1}^{n}\left\|\left(\varphi_{\bar{x}_{r}}^{h}\right)_{x_{r}, m_{r}}\right\|_{L_{2}\left(\widetilde{\Omega}_{h}\right)}\right] .
\end{aligned}
$$

Here $M_{1}$ does not depend on $\tau, h, \varphi^{h}(x), \psi^{h}(x)$, and $f_{k}^{h}(x), 0 \leq k \leq N-1$.

The proof of Theorem 2.8 is based on the abstract Theorem 2.2, and the symmetry properties of the difference operator $A_{h}^{x}$ defined by the formula (2.56).

Theorem 2.9. Let $\tau$ and $|h|$ be sufficiently small numbers. Then the solutions of the difference schemes (2.56) for $j=l+1$ satisfy the following stability estimates:

$$
\begin{aligned}
\max _{0 \leq k \leq N} & \left\|u_{k}^{h}\right\|_{L_{2}\left(\tilde{\Omega}_{h}\right)} \\
\leq & M_{1}\left[\max _{0 \leq k \leq N-1}\left\|f_{k}^{h}\right\|_{L_{2}\left(\tilde{\Omega}_{h}\right)}+\left\|\psi^{h}\right\|_{L_{2}\left(\tilde{\Omega}_{h}\right)}+\sum_{r=1}^{n}\left\|\varphi_{x_{r}, \bar{m}_{r}}^{h}\right\|_{L_{2}\left(\tilde{\Omega}_{h}\right)}\right], \\
\max _{1 \leq k \leq N}\left\|\tau^{-1}\left(u_{k}^{h}-u_{k-1}^{h}\right)\right\|_{L_{2}\left(\tilde{\Omega}_{h}\right)} & \\
\leq & M_{1}\left[\max _{0 \leq k \leq N-1} \sum_{r=1}^{n}\left\|\left(f_{k}^{h}\right)_{x_{r}, \bar{m}_{r}}\right\|_{L_{2}\left(\tilde{\Omega}_{h}\right)}+\sum_{r=1}^{n}\left\|\psi_{x_{r}, \bar{m}_{r}}^{h}\right\|_{L_{2}\left(\tilde{\Omega}_{h}\right)}+\sum_{r=1}^{n}\left\|\left(\varphi_{\bar{x}_{r}}^{h}\right)_{x_{r}, m_{r}}\right\|_{L_{2}\left(\tilde{\Omega}_{h}\right)}\right], \\
\max _{1 \leq k \leq N-1}\left\|\tau^{-2}\left(u_{k+1}^{h}-2 u_{k}^{h}+u_{k-1}^{h}\right)\right\|_{L_{2}\left(\tilde{\Omega}_{h}\right)} & {\left[M _ { 1 } \left[\max _{1 \leq k \leq N-1} \tau^{-1} \sum_{r=1}^{n}\left\|\left(f_{k}^{h}\right)_{x_{r}, \bar{m}_{r}}-\left(f_{k-1}^{h}\right)_{x_{r}, \bar{m}_{r}}\right\|_{L_{2}\left(\bar{\Omega}_{h}\right)}+\left.\sum_{r=1}^{n}\left\|\left(f_{0}^{h}\right)_{x_{r}, \bar{m}_{r}}\right\|\right|_{L_{2}\left(\tilde{\Omega}_{h}\right)}\right.\right.} \\
& \left.\quad+\sum_{r=1}^{n}\left\|\left(\psi_{\bar{x}_{r}}^{h}\right)_{x_{r}, m_{r}}\right\|_{L_{2}\left(\tilde{\Omega}_{h}\right)}+\sum_{r=1}^{n}\left\|\left(\left(\varphi_{\bar{x}_{r}}^{h}\right)_{x_{r}}\right)_{x_{r}, m_{r}}\right\|_{L_{2}\left(\tilde{\Omega}_{h}\right)}\right] .
\end{aligned}
$$

Here $M_{1}$ does not depend on $\tau, h, \varphi^{h}(x), \psi^{h}(x)$, and $f_{k}^{h}(x), 0 \leq k \leq N-1$.

The proof of Theorem 2.9 is based on the abstract Theorem 2.5, and the symmetry properties of the difference operator $A_{h}^{x}$ defined by the formula (2.56).

Note that in a similar manner one can construct the difference schemes of a high order of accuracy with respect to one variable for approximate solutions of the boundary value problem (1.17). Abstract Theorems 2.2 and 2.5 permit us to obtain the stability estimates for the solutions of these difference schemes. 


\section{The high order of accuracy difference schemes generated by Taylor's decomposition of function on the three points}

We consider again the initial value problem (1.1). For the construction of the two-step difference schemes of an arbitrary high order of accuracy for the approximate solutions of the initial value problem (1.1), we consider again a uniform grid space

$$
[0, T]_{\tau}=\left\{t_{k}=k \tau, k=0,1, \ldots, N, N \tau=T\right\} .
$$

The function $v(t)(0 \leq t \leq T)$ has a $(2 l+2 j+2)$ th continuous derivative and $t_{k-1}, t_{k}$, $t_{k+1} \in[0, T]_{\tau}$. Then we have the following Taylor decomposition on the three points (see $[6]):$

$$
\begin{aligned}
v\left(t_{k+1}\right) & -2 v\left(t_{k}\right)+v\left(t_{k-1}\right)-\sum_{s=1}^{l} \alpha_{s} v^{(2 s)}\left(t_{k}\right) \tau^{2 s}-\sum_{s=1}^{j} \eta_{s}\left(v^{(2 s)}\left(t_{k-1}\right)+v^{(2 s)}\left(t_{k+1}\right)\right) \tau^{2 s} \\
& =o\left(\tau^{2 l+2 j+2}\right)
\end{aligned}
$$

where $\eta_{m}, m=1, \ldots, j$, is the solution of system

$$
\begin{gathered}
\eta_{s}=\frac{1}{(2 s) !}-\sum_{m=1}^{s-1} \frac{\eta_{m}}{(2 s-2 m) !} \quad \text { for any } s, l+1 \leq s \leq j, \\
\sum_{m=1}^{j} \frac{(2 s) ! \eta_{m}}{(2 s-2 m) !}=1 \quad \text { for any } s, j+1 \leq s \leq l+j, \\
\alpha_{1}=1-2 \eta_{1}, \quad \alpha_{s}=\left(\frac{1}{(2 s) !}-\eta_{s}-\sum_{m=1}^{s-1} \eta_{m} \frac{1}{(2(s-m)) !}\right) 2 \quad \text { for any } s, 2 \leq s \leq l .
\end{gathered}
$$

Suppose further that the function $v(t)(0 \leq t \leq T)$ has a $(2 l+2 j+1)$ th continuous derivative. Then we have the following Taylor decomposition on the two points (see [6]):

$$
v(\tau)-v(0)+\sum_{s=1}^{2 j} \delta_{s} v^{(s)}(\tau) \tau^{s}-\sum_{s=1}^{2 l} \rho_{s} v^{(s)}(0) \tau^{s}=o\left(\tau^{2 l+2 j+1}\right)
$$

where

$$
\begin{aligned}
\rho_{s} & =\frac{(2 l+2 j-s) !(2 l) !}{(2 l+2 j) ! s !(2 l-s) !} \quad \text { for any } s, 1 \leq s \leq 2 l, \\
\delta_{s} & =\frac{(2 l+2 j-s) !(2 j) !(-1)^{s}}{(2 l+2 j) ! s !(2 j-s) !} \quad \text { for any } s, 1 \leq s \leq 2 j .
\end{aligned}
$$

Now, we will consider the applications of the Taylor decomposition (3.2) of function $v(t)$ on the three points and Taylor's decomposition (3.4) of function $v(t)$ on the two points to approximate solutions of the initial value problem (1.1). From (3.2) and (3.4) it is clear that for the approximate solution of the problem (1.1), it is necessary to find $v^{(j)}(\tau)$ for any $s, 1 \leq s \leq 2 l, v^{(2 s)}\left(t_{k}\right)$ for any $s, 1 \leq s \leq l$, and $v^{(2 s)}\left(t_{k-1}\right), v^{(2 s)}\left(t_{k+1}\right)$ 
204 High order of accuracy difference schemes for HDE

for any $s, 1 \leq s \leq j$. Using the equation

$$
v^{\prime \prime}(t)=-A v(t)+f(t)
$$

we obtain

$$
\begin{gathered}
v^{(2 n)}(t)=(-A)^{n} v(t)+\sum_{\lambda=1}^{n}(-A)^{n-\lambda} f^{(2 \lambda-2)}(t), \quad n=2, \ldots, \\
v^{(2 n+1)}(t)=(-A)^{n} v^{\prime}(t)+\sum_{\lambda=1}^{n}(-A)^{n-\lambda} f^{(2 \lambda-1)}(t), \quad n=1, \ldots
\end{gathered}
$$

Suppose further that the function $v(t)(0 \leq t \leq T)$ has a $(2 l+2 j-2 m+2)$ th continuous derivative. Then by Theorem 1.3, we have the following Taylor decomposition on the two points:

$$
v(\tau)-v(0)+\sum_{s=1}^{2 l-2[m / 2]} a_{s} v^{(s)}(\tau) \tau^{s}-\sum_{s=1}^{2 j-2 m+2[m / 2]+1} b_{s} v^{(s)}(0) \tau^{s}=o\left(\tau^{2 l+2 j-2 m+2}\right),
$$

where

$$
\begin{gathered}
a_{s}=\frac{(2 l+2 j-2 m+1-s) !(2 l-2[m / 2]) !(-1)^{s}}{(2 l+2 j+1-2 m) ! s !(2 p-2[m / 2]-s) !} \text { for any } s, 1 \leq s \leq 2 l-2\left[\frac{m}{2}\right], \\
b_{s}=\frac{(2 l+2 j+1-2 m-s) !(2 j-2 m+2[m / 2]+1) !}{(2 l+2 j+1-2 m) ! s !(2 j-2 m+2[m / 2]+1-s) !} \\
\quad \text { for any } s, 1 \leq s \leq 2 j-2 m+2\left[\frac{m}{2}\right]+1,
\end{gathered}
$$

where $[a]$ denotes the integer part of the number $a$. Further using the formulas (3.7), (3.8), and (3.9), we can write

$$
\begin{aligned}
v^{\prime}(\tau) & -v^{\prime}(0)+\sum_{n=1}^{l-[m / 2]} a_{2 n}(-A)^{n} v^{\prime}(\tau) \tau^{2 n}+\sum_{n=1}^{l-[m / 2]} a_{2 n} \sum_{\lambda=1}^{n}(-A)^{n-\lambda} f^{(2 \lambda-1)}(\tau) \tau^{2 n} \\
& +\sum_{n=1}^{l-[m / 2]} a_{2 n-1}(-A)^{n} v(\tau) \tau^{2 n-1}+\sum_{n=1}^{l-[m / 2]} a_{2 n-1} \sum_{\lambda=1}^{n}(-A)^{n-\lambda} f^{(2 \lambda-2)}(\tau) \tau^{2 n-1} \\
& -\sum_{n=1}^{j-m+[m / 2]} b_{2 n}(-A)^{n} v^{\prime}(0) \tau^{2 n}-\sum_{n=1}^{j-m+[m / 2]} b_{2 n} \sum_{\lambda=1}^{n}(-A)^{n-\lambda} f^{(2 \lambda-1)}(0) \tau^{2 n} \\
& -\sum_{n=1}^{j-m+[m / 2]+1} b_{2 n-1}(-A)^{n} v(0) \tau^{2 n-1}-\sum_{n=1}^{j-m+[m / 2]+1} b_{2 n-1} \sum_{\lambda=1}^{n}(-A)^{n-\lambda} f^{(2 \lambda-2)}(0) \tau^{2 n-1} \\
= & o\left(\tau^{2 l+2 j-2 m+2}\right), \quad 0 \leq m \leq j-1 .
\end{aligned}
$$


From the last formula it follows that

$$
\begin{aligned}
(I+ & \left.\sum_{n=1}^{l-[m / 2]} a_{2 n}(-A)^{n} \tau^{2 n}\right) v^{\prime}(\tau) \\
= & \left(I+\sum_{n=1}^{j-m+[m / 2]} b_{2 n}(-A)^{n} \tau^{2 n}\right) v^{\prime}(0)-\sum_{n=1}^{l-[m / 2]} a_{2 n-1}(-A)^{n} v(\tau) \tau^{2 n-1} \\
& +\sum_{n=1}^{j-m+[m / 2]+1} b_{2 n-1}(-A)^{n} v(0) \tau^{2 n-1}-\sum_{n=1}^{l-[m / 2]} a_{2 n} \sum_{\lambda=1}^{n}(-A)^{n-\lambda} f^{(2 \lambda-1)}(\tau) \tau^{2 n} \\
& \quad-\sum_{n=1}^{l-[m / 2]} a_{2 n-1} \sum_{\lambda=1}^{n}(-A)^{n-\lambda} f^{(2 \lambda-2)}(\tau) \tau^{2 n-1}+\sum_{n=1}^{j-m+[m / 2]} b_{2 n} \sum_{\lambda=1}^{n}(-A)^{n-\lambda} f^{(2 \lambda-1)}(0) \tau^{2 n} \\
& +\sum_{n=1}^{j-m+[m / 2]+1} b_{2 n-1} \sum_{\lambda=1}^{n}(-A)^{n-\lambda} f^{(2 \lambda-2)}(0) \tau^{2 n-1}+o\left(\tau^{2 l+2 j-2 m+2}\right), \quad 0 \leq m \leq j-1 .
\end{aligned}
$$

Suppose further that the operator $\left(I+\sum_{n=1}^{l-[m / 2]} a_{2 n}(-A)^{n} \tau^{2 n}\right)$ has a bounded inverse. Then

$$
\begin{aligned}
v^{\prime}(\tau)= & \left(I+\sum_{n=1}^{l-[m / 2]} a_{2 n}(-A)^{n} \tau^{2 n}\right)^{-1} \\
& \times\left\{\left(I+\sum_{n=1}^{j-m+[m / 2]} b_{2 n}(-A)^{n} \tau^{2 n}\right) v^{\prime}(0)-\sum_{n=1}^{l-[m / 2]} a_{2 n-1}(-A)^{n} v(\tau) \tau^{2 n-1}\right. \\
& +\sum_{n=1}^{j-m+[m / 2]+1} b_{2 n-1}(-A)^{n} v(0) \tau^{2 n-1}-\sum_{n=1}^{l-[m / 2]} a_{2 n} \sum_{\lambda=1}^{n}(-A)^{n-\lambda} f^{(2 \lambda-1)}(\tau) \tau^{2 n} \\
& \quad-\sum_{n=1}^{l-[m / 2]} a_{2 n-1} \sum_{\lambda=1}^{n}(-A)^{n-\lambda} f^{(2 \lambda-2)}(\tau) \tau^{2 n-1} \\
& +\sum_{n=1}^{j-m+[m / 2]} b_{2 n} \sum_{\lambda=1}^{n}(-A)^{n-\lambda} f^{(2 \lambda-1)}(0) \tau^{2 n} \\
& \left.+\sum_{n=1}^{j-m+[m / 2]+1} b_{2 n-1} \sum_{\lambda=1}^{n}(-A)^{n-\lambda} f^{(2 \lambda-2)}(0) \tau^{2 n-1}\right\} \\
+ & o\left(\tau^{2 l+2 j-2 m+2}\right), \quad 0 \leq m \leq j-1 .
\end{aligned}
$$


Now, using the formulas (3.2), (3.4), (3.7), (3.8), and (3.13), we obtain the difference schemes of a $(2 l+2 j)$ th order of accuracy

$$
\begin{aligned}
& \frac{u_{k+1}-2 u_{k}+u_{k-1}}{\tau^{2}}-\sum_{s=1}^{l} \alpha_{s}(-A)^{s} \tau^{2 s-2} u_{k}-\sum_{s=1}^{j} \eta_{s}(-A)^{s} \tau^{2 s-2}\left(u_{k-1}+u_{k+1}\right)=f_{k}^{j, l}, \\
& f_{k}^{j, l}=\sum_{s=1}^{j} \eta_{s} \sum_{\lambda=1}^{s}(-A)^{s-\lambda}\left(f^{(2 \lambda-2)}\left(t_{k+1}\right)+f^{(2 \lambda-2)}\left(t_{k-1}\right)\right) \tau^{2 s-2} \\
& +\sum_{s=1}^{l} \alpha_{s} \sum_{\lambda=1}^{s}(-A)^{s-\lambda} f^{(2 \lambda-2)}\left(t_{k}\right) \tau^{2 s-2}, \quad 1 \leq k \leq N-1, \quad u_{0}=v_{0}, \\
& \frac{u_{1}-u_{0}}{\tau}-\sum_{n=1}^{l} \rho_{2 n}(-A)^{n} \tau^{2 n-1} v(0)-\sum_{n=1}^{l} \rho_{2 n-1}(-A)^{n-1} \tau^{2 n-2} v^{\prime}(0) \\
& +\sum_{n=1}^{j} \delta_{2 n}(-A)^{n} \tau^{2 n-1} u_{1}+\sum_{m=1}^{j} \delta_{2 m-1}(-A)^{m} \tau^{2 m-2} \times\left(I+\sum_{n=1}^{l-[m / 2]} a_{2 n}(-A)^{n} \tau^{2 n}\right)^{-1} \\
& \times\left\{\left(I+\sum_{n=1}^{j-m+[m / 2]} b_{2 n}(-A)^{n} \tau^{2 n}\right) v^{\prime}(0)-\sum_{n=1}^{l-[m / 2]} a_{2 n-1}(-A)^{n} \tau^{2 n-1} u_{1}\right. \\
& \left.+\sum_{n=1}^{j-m+[m / 2]+1} b_{2 n-1}(-A)^{n} \tau^{2 n-1} v(0)\right\} \\
& =\sum_{n=1}^{l} \rho_{2 n}(-A)^{n} \tau^{2 n-1} \sum_{\lambda=1}^{n}(-A)^{n-\lambda} f^{(2 \lambda-2)}(0) \\
& +\sum_{n=1}^{l} \rho_{2 n-1}(-A)^{n-1} \tau^{2 n-2} \sum_{\lambda=1}^{n}(-A)^{n-\lambda} f^{(2 \lambda-1)}(0) \\
& -\sum_{n=1}^{j} \delta_{2 n}(-A)^{n} \tau^{2 n-1} \sum_{\lambda=1}^{n}(-A)^{n-\lambda} f^{(2 \lambda-2)}(\tau) \\
& -\sum_{n=1}^{j} \delta_{2 n-1}(-A)^{n} \tau^{2 n-2} \sum_{\lambda=1}^{n-1}(-A)^{n-\lambda} f^{(2 \lambda-1)}(\tau) \\
& -\sum_{m=1}^{j} \delta_{2 m-1}(-A)^{m} \tau^{2 m-2}\left(I+\sum_{n=1}^{l-[m / 2]} a_{2 n}(-A)^{n} \tau^{2 n}\right)^{-1} \\
& \times\left\{-\sum_{n=1}^{l-[m / 2]} a_{2 n} \sum_{\lambda=1}^{n}(-A)^{n-\lambda} f^{(2 \lambda-1)}(\tau) \tau^{2 n}-\sum_{n=1}^{l-[m / 2]} a_{2 n-1} \sum_{\lambda=1}^{n}(-A)^{n-\lambda} f^{(2 \lambda-2)}(\tau) \tau^{2 n-1}\right. \\
& +\sum_{n=1}^{j-m+[m / 2]} b_{2 n} \sum_{\lambda=1}^{n}(-A)^{n-\lambda} f^{(2 \lambda-1)}(0) \tau^{2 n} \\
& \left.+\sum_{n=1}^{j-m+[m / 2]+1} b_{2 n-1} \sum_{\lambda=1}^{n}(-A)^{n-\lambda} f^{(2 \lambda-2)}(0) \tau^{2 n-1}\right\}
\end{aligned}
$$

for the approximate solution of the initial value problem (1.1). 
Suppose that the operator

$$
\begin{aligned}
& \left(I+\sum_{n=1}^{j} \delta_{2 n}(-A)^{n} \tau^{2 n}-\sum_{m=1}^{j} \delta_{2 m-1}(-A)^{m} \tau^{2 m-1}\left(I+\sum_{n=1}^{l-[m / 2]} a_{2 n}(-A)^{n} \tau^{2 n}\right)^{-1}\right. \\
& \left.\times \sum_{n=1}^{l-[m / 2]} a_{2 n-1}(-A)^{n} \tau^{2 n-1}\right)
\end{aligned}
$$

has a bounded inverse. Suppose further that the operator $\left(I-\sum_{s=1}^{j} \eta_{s}(-A)^{s} \tau^{2 s}\right)$ has a bounded inverse and

$$
I-\frac{1}{4}\left(2 I+\sum_{s=1}^{l} \alpha_{s}(-A)^{s} \tau^{2 s}\right)^{2}\left(I-\sum_{s=1}^{j} \eta_{s}(-A)^{s} \tau^{2 s}\right)^{-2} \geq 0
$$

This problem is uniquely solvable, and the following formula holds:

$$
\begin{aligned}
& u_{k}=\left(\widetilde{R}_{j, l}(-i \tau A)-\widetilde{R}_{j, l}(i \tau A)\right)^{-1} \widetilde{R}_{j, l}(i \tau A) \widetilde{R}_{j, l}(-i \tau A)\left(\widetilde{R}_{j, l}^{k-1}(i \tau A)-\widetilde{R}_{j, l}^{k-1}(-i \tau A)\right) u_{0} \\
&+\left(\widetilde{R}_{j, l}(-i \tau A)-\widetilde{R}_{j, l}(i \tau A)\right)^{-1}\left(\widetilde{R}_{j, l}^{k}(-i \tau A)-\widetilde{R}_{j, l}^{k}(i \tau A)\right) u_{1} \\
&+\sum_{j=1}^{k}\left(\widetilde{R}_{j, l}(-i \tau A)-\widetilde{R}_{j, l}(i \tau A)\right)_{j, l}^{-1} \widetilde{R}_{j, l}(i \tau A) \widetilde{R}_{j, l}(-i \tau A) \\
& \times\left(\widetilde{R}_{j, l}^{k-m}(-i \tau A)-\widetilde{R}_{j, l}^{k-m}(i \tau A)\right)\left(I-\sum_{s=1}^{j} \eta_{s}(-A)^{s} \tau^{2 s}\right)^{-1} f_{m}^{j, l}, \quad 2 \leq k \leq N, \\
& u_{1}=\widetilde{c}_{\tau} v(0)+\widetilde{s}_{\tau} v^{\prime}(0)+\tau f_{0}^{j, l} .
\end{aligned}
$$

Here

$$
\begin{aligned}
\widetilde{R}_{j, l}( \pm i \tau A)= & \frac{1}{2}\left(2 I+\sum_{s=1}^{l} \alpha_{s}(-A)^{s} \tau^{2 s}\right)\left(I-\sum_{s=1}^{j} \eta_{s}(-A)^{s} \tau^{2 s}\right)^{-1} \\
& \pm i \sqrt{I-\frac{1}{4}\left(2 I+\sum_{s=1}^{l} \alpha_{s}(-A)^{s} \tau^{2 s}\right)^{2}\left(I-\sum_{s=1}^{j} \eta_{s}(-A)^{s} \tau^{2 s}\right)^{-2}}
\end{aligned}
$$


208 High order of accuracy difference schemes for HDE

$$
\begin{aligned}
& \tilde{c}_{\tau}=\left(I+\sum_{n=1}^{j} \delta_{2 n}(-A)^{n} \tau^{2 n}-\sum_{m=1}^{j} \delta_{2 m-1}(-A)^{m} \tau^{2 m-1}\right. \\
& \left.\times\left(I+\sum_{n=1}^{l-[m / 2]} a_{2 n}(-A)^{n} \tau^{2 n}\right)^{-1} \sum_{n=1}^{l-[m / 2]} a_{2 n-1}(-A)^{n} \tau^{2 n-1}\right)^{-1} \\
& \times\left\{\sum_{n=1}^{l} \rho_{2 n}(-A)^{n} \tau^{2 n-1}+\sum_{m=1}^{j} \delta_{2 m-1}(-A)^{m} \tau^{2 m-2}\right. \\
& \left.\times\left(I+\sum_{n=1}^{l-[m / 2]} a_{2 n}(-A)^{n} \tau^{2 n}\right)^{-1} \sum_{n=1}^{j-m+[m / 2]+1} b_{2 n-1}(-A)^{n} \tau^{2 n-1}\right\}, \\
& \widetilde{\boldsymbol{s}}_{\tau}=\left(I+\sum_{n=1}^{j} \delta_{2 n}(-A)^{n} \tau^{2 n}-\sum_{m=1}^{j} \delta_{2 m-1}(-A)^{m} \tau^{2 m-1}\right. \\
& \left.\times\left(I+\sum_{n=1}^{l-[m / 2]} a_{2 n}(-A)^{n} \tau^{2 n}\right)^{-1} \sum_{n=1}^{l-[m / 2]} a_{2 n-1}(-A)^{n} \tau^{2 n-1}\right)^{-1} \\
& \times\left\{\sum_{n=1}^{l} \rho_{2 n-1}(-A)^{n-1} \tau^{2 n-2}+\sum_{m=1}^{j} \delta_{2 m-1}(-A)^{m} \tau^{2 m-2}\right. \\
& \left.\times\left(I+\sum_{n=1}^{l-[m / 2]} a_{2 n}(-A)^{n} \tau^{2 n}\right)^{-1}\left(I+\sum_{n=1}^{j-m+[m / 2]} b_{2 n}(-A)^{n} \tau^{2 n}\right)\right\}, \\
& f_{0}^{j, l}=\sum_{n=1}^{l} \rho_{2 n}(-A)^{n} \tau^{2 n-1} \sum_{\lambda=1}^{n}(-A)^{n-\lambda} f^{(2 \lambda-2)}(0) \\
& +\sum_{n=1}^{l} \rho_{2 n-1}(-A)^{n-1} \tau^{2 n-2} \sum_{\lambda=1}^{n}(-A)^{n-\lambda} f^{(2 \lambda-1)}(0) \\
& -\sum_{n=1}^{j} \delta_{2 n}(-A)^{n} \tau^{2 n-1} \sum_{\lambda=1}^{n}(-A)^{n-\lambda} f^{(2 \lambda-2)}(\tau) \\
& -\sum_{n=1}^{j} \delta_{2 n-1}(-A)^{n} \tau^{2 n-2} \sum_{\lambda=1}^{n-1}(-A)^{n-\lambda} f^{(2 \lambda-1)}(\tau) \\
& -\sum_{m=1}^{j} \delta_{2 m-1}(-A)^{m} \tau^{2 m-2}\left(I+\sum_{n=1}^{l-[m / 2]} a_{2 n}(-A)^{n} \tau^{2 n}\right)^{-1} \\
& \times\left\{-\sum_{n=1}^{l-[m / 2]} a_{2 n} \sum_{\lambda=1}^{n}(-A)^{n-\lambda} f^{(2 \lambda-1)}(\tau) \tau^{2 n}\right. \\
& -\sum_{n=1}^{l-[m / 2]} a_{2 n-1} \sum_{\lambda=1}^{n}(-A)^{n-\lambda} f^{(2 \lambda-2)}(\tau) \tau^{2 n-1} \\
& +\sum_{n=1}^{j-m+[m / 2]} b_{2 n} \sum_{\lambda=1}^{n}(-A)^{n-\lambda} f^{(2 \lambda-1)}(0) \tau^{2 n} \\
& \left.+\sum_{n=1}^{j-m+[m / 2]+1} b_{2 n-1} \sum_{\lambda=1}^{n}(-A)^{n-\lambda} f^{(2 \lambda-2)}(0) \tau^{2 n-1}\right\} \text {. }
\end{aligned}
$$


From the formula (3.17) it follows that the investigation of the stability of difference schemes (3.14) relies in an essential manner on a number of properties of the powers of the operator $\widetilde{R}_{j, l}( \pm i \tau A)$. We were not able to obtain the estimates for powers of the operator $\widetilde{R}_{j, l}( \pm i \tau A)$ in the general cases of numbers $j$ and $l$.

Theorem 3.1. Suppose that $u_{0} \in D(A), u_{1}-u_{0} \in D\left(A^{1 / 2}\right)$. Then for the solution of the two-step difference schemes (3.14) for $l=0$ the following stability inequalities hold:

$$
\begin{aligned}
& \max _{0 \leq k \leq N}\left\|u_{k}\right\|_{H} \\
& \quad \leq M\left[\max _{1 \leq k \leq N-1}\left\|A^{-1} f_{k}^{j, l}\right\|_{H}+\left\|A^{-1 / 2} \tau^{-1}\left(u_{1}-u_{0}\right)\right\|_{H}+\left\|u_{0}\right\|_{H}\right], \\
& \max _{1 \leq k \leq N}\left\|\tau^{-1}\left(u_{k}-u_{k-1}\right)\right\|_{H} \\
& \quad \leq M\left[\max _{1 \leq k \leq N-1}\left\|f_{k}^{j, l}\right\|_{H}+\left\|\tau^{-1}\left(u_{1}-u_{0}\right)\right\|_{H}+\left\|A^{1 / 2} u_{0}\right\|_{H}\right], \\
& \max _{1 \leq k \leq N-1}\left\|\tau^{-2}\left(u_{k+1}-2 u_{k}+u_{k-1}\right)\right\| \|_{H} \\
& \quad \leq M\left[\max _{2 \leq k \leq N-1}\left\|\tau^{-1}\left(f_{k}^{j, l}-f_{k-1}^{j, l}\right)\right\|_{H}+\left\|f_{1}^{j, l}\right\|_{H}+\left\|A^{1 / 2} \tau^{-1}\left(u_{1}-u_{0}\right)\right\|_{H}+\left\|A u_{0}\right\|_{H}\right],
\end{aligned}
$$

where $M$ does not depend on $\tau, f_{k}^{j, l}, 1 \leq k \leq N-1$, and $u_{0}, u_{1}$.

The proof of Theorem 3.1 follows the scheme of the proof of Theorem 2.1, and relies on the formula (3.17) and on the estimates

$$
\begin{gathered}
\left\|\widetilde{R}_{j, l}( \pm i \tau A)\right\|_{H \rightarrow H} \leq 1, \\
\left\|\tau A^{1 / 2}\left(I-\widetilde{R}_{j, l}^{2}(i \tau A)\right)^{-1} \widetilde{R}_{j, l}(i \tau A)\right\|_{H \rightarrow H} \leq M, \\
\left\|\left(I-\sum_{s=1}^{j} \eta_{s}(-A)^{s} \tau^{2 s}\right)^{-1}\right\|_{H \rightarrow H} \leq M .
\end{gathered}
$$

Remark 3.2. Theorem 3.1 actually holds in the general cases of numbers $j$ and $l$ under the following assumption:

$$
\tau\|A\|_{H \rightarrow H} \longrightarrow 0 \quad \text { when } \tau \longrightarrow 0 .
$$


Remark 3.3. Applying the formulas (2.37), (2.41), (2.44), (3.2), (3.7), and (3.13), we can construct the difference schemes of a $(2 l+2 j)$ th order of accuracy

$$
\begin{gathered}
\frac{u_{k+1}-2 u_{k}+u_{k-1}}{\tau^{2}}-\sum_{s=1}^{l} \alpha_{s}(-A)^{s} \tau^{2 s-2} u_{k}-\sum_{s=1}^{j} \eta_{s}(-A)^{s} \tau^{2 s-2}\left(u_{k-1}+u_{k+1}\right)=f_{k}^{j, l}, \\
f_{k}^{j, l}=\sum_{s=1}^{j} \eta_{s} \sum_{\lambda=1}^{s}(-A)^{s-\lambda}\left(f^{(2 \lambda-2)}\left(t_{k+1}\right)+f^{(2 \lambda-2)}\left(t_{k-1}\right)\right) \tau^{2 s-2} \\
+\sum_{s=1}^{l} \alpha_{s} \sum_{\lambda=1}^{s}(-A)^{s-\lambda} f^{(2 \lambda-2)}\left(t_{k}\right) \tau^{2 s-2}, \quad 1 \leq k \leq N-1, \quad u_{0}=v_{0}, \\
\tau^{-1}\left(u_{1}-u_{0}\right)=\tau^{-1}\left(\frac{R_{2 l, 2 j}(i \tau B)+R_{2 l, 2 j}(-i \tau B)}{2}-I\right) v_{0} \\
+\tau^{-1} B^{-1} \frac{R_{2 l, 2 j}(i \tau B)-R_{2 l, 2 j}(-i \tau B)}{2 i} v^{\prime}(0)+f_{0}^{j, l}
\end{gathered}
$$

for the approximate solution of the initial value problem (1.1). In a similar manner one can obtain the stability inequalities for the solution of the two-step difference schemes (3.22).

Now, the abstract Theorem 3.1 is applied in the investigation of difference schemes of higher order of accuracy with respect to one variable for approximate solutions of the mixed boundary value problem (1.19). The first step of discretization of problem (1.19) is given above. Suppose that the operator

$$
\begin{aligned}
& \left(I+\sum_{n=1}^{j} \delta_{2 n}(-A)^{n} \tau^{2 n}-\sum_{m=1}^{j} \delta_{2 m-1}(-A)^{m} \tau^{2 m-1}\left(I+\sum_{n=1}^{l-[m / 2]} a_{2 n}(-A)^{n} \tau^{2 n}\right)^{-1}\right. \\
& \left.\quad \times \sum_{n=1}^{l-[m / 2]} a_{2 n-1}(-A)^{n} \tau^{2 n-1}\right)
\end{aligned}
$$

has a bounded inverse. Suppose further that the operator $\left(I-\sum_{s=1}^{j} \eta_{s}\left(-A_{h}^{x}\right)^{s} \tau^{2 s}\right)$ has a bounded inverse and

$$
I-\frac{1}{4}\left(2 I+\sum_{s=1}^{l} \alpha_{s}\left(-A_{h}^{x}\right)^{s} \tau^{2 s}\right)^{2}\left(I-\sum_{s=1}^{j} \eta_{s}\left(-A_{h}^{x}\right)^{s} \tau^{2 s}\right)^{-2} \geq 0
$$

Then in the second step we replace problem (2.56) by the difference schemes (3.14):

$$
\begin{gathered}
\tau^{-2}\left(u_{k+1}^{h}(x)-2 u_{k}^{h}(x)+u_{k-1}^{h}(x)\right)+\widetilde{A_{h}^{x}} u_{k}^{h}(x)+\widetilde{A A_{h}^{x}}\left(u_{k+1}^{h}(x)+u_{k-1}^{h}(x)\right)=f_{k}^{h}(x), \\
t_{k}=k \tau, \quad 1 \leq k \leq N-1, \quad x \in \Omega_{h}, \\
\widetilde{A_{h}^{x}}=-\sum_{s=1}^{l} \alpha_{s}\left(-A_{h}^{x}\right)^{s} \tau^{2 s-2}, \quad \widetilde{A A_{h}^{x}}=-\sum_{s=1}^{j} \eta_{s}\left(-A_{h}^{x}\right)^{s} \tau^{2 s-2},
\end{gathered}
$$




$$
\begin{aligned}
& f_{k}^{h}(x)=\sum_{s=1}^{j} \eta_{s} \sum_{\lambda=1}^{s}\left(-A_{h}^{x}\right)^{s-\lambda}\left(f^{(2 \lambda-2)}\left(t_{k+1}, x\right)+f^{(2 \lambda-2)}\left(t_{k-1}, x\right)\right) \tau^{2 s-2} \\
& +\sum_{s=1}^{l} \alpha_{i} \sum_{\lambda=1}^{s}\left(-A_{h}^{x}\right)^{s-\lambda} f^{(2 \lambda-2)}\left(t_{k}, x\right) \tau^{2 s-2}, \quad 1 \leq k \leq N-1, \\
& u_{1}^{h}(x)=\tilde{c}_{\tau} \varphi^{h}(x)+\tilde{s}_{\tau} \psi^{h}(x)+\tau f_{0}^{h}(x), \quad x \in \Omega_{h}, \\
& \tilde{c}_{\tau}=\left(I+\sum_{n=1}^{j} \delta_{2 n}\left(-A_{h}^{x}\right)^{n} \tau^{2 n}-\sum_{m=1}^{j} \delta_{2 m-1}\left(-A_{h}^{x}\right)^{m} \tau^{2 m-1}\right. \\
& \left.\times\left(I+\sum_{n=1}^{l-[m / 2]} a_{2 n}\left(-A_{h}^{x}\right)^{n} \tau^{2 n}\right)^{-1} \sum_{n=1}^{l-[m / 2]} a_{2 n-1}\left(-A_{h}^{x}\right)^{n} \tau^{2 n-1}\right)^{-1} \\
& \times\left\{\sum_{n=1}^{l} \rho_{2 n}\left(-A_{h}^{x}\right)^{n} \tau^{2 n-1}+\sum_{m=1}^{j} \delta_{2 m-1}\left(-A_{h}^{x}\right)^{m} \tau^{2 m-2}\right. \\
& \left.\times\left(I+\sum_{n=1}^{l-[m / 2]} a_{2 n}\left(-A_{h}^{x}\right)^{n} \tau^{2 n}\right)^{-1} \sum_{n=1}^{j-m+[m / 2]+1} b_{2 n-1}\left(-A_{h}^{x}\right)^{n} \tau^{2 n-1}\right\}, \\
& \widetilde{\mathcal{s}}_{\tau}=\left(I+\sum_{n=1}^{j} \delta_{2 n}\left(-A_{h}^{x}\right)^{n} \tau^{2 n}-\sum_{m=1}^{j} \delta_{2 m-1}\left(-A_{h}^{x}\right)^{m} \tau^{2 m-1}\right. \\
& \left.\times\left(I+\sum_{n=1}^{l-[m / 2]} a_{2 n}\left(-A_{h}^{x}\right)^{n} \tau^{2 n}\right)^{-1} \sum_{n=1}^{l-[m / 2]} a_{2 n-1}\left(-A_{h}^{x}\right)^{n} \tau^{2 n-1}\right)^{-1} \\
& \times\left\{\sum_{n=1}^{l} \rho_{2 n-1}\left(-A_{h}^{x}\right)^{n-1} \tau^{2 n-2}+\sum_{m=1}^{j} \delta_{2 m-1}\left(-A_{h}^{x}\right)^{m} \tau^{2 m-2}\right. \\
& \left.\times\left(I+\sum_{n=1}^{l-[m / 2]} a_{2 n}\left(-A_{h}^{x}\right)^{n} \tau^{2 n}\right)^{-1}\left(I+\sum_{n=1}^{j-m+[m / 2]} b_{2 n}\left(-A_{h}^{x}\right)^{n} \tau^{2 n}\right)\right\}, \\
& f_{0}^{h}(x)=\sum_{n=1}^{l} \rho_{2 n}\left(-A_{h}^{x}\right)^{n} \tau^{2 n-1} \sum_{\lambda=1}^{n}\left(-A_{h}^{x}\right)^{n-\lambda} f^{(2 \lambda-2)}(0, x) \\
& +\sum_{n=1}^{l} \rho_{2 n-1}\left(-A_{h}^{x}\right)^{n-1} \tau^{2 n-2} \sum_{\lambda=1}^{n}\left(-A_{h}^{x}\right)^{n-\lambda} f^{(2 \lambda-1)}(0, x) \\
& -\sum_{n=1}^{j} \delta_{2 n}\left(-A_{h}^{x}\right)^{n} \tau^{2 n-1} \sum_{\lambda=1}^{n}\left(-A_{h}^{x}\right)^{n-\lambda} f^{(2 \lambda-2)}(\tau, x) \\
& -\sum_{n=1}^{j} \delta_{2 n-1}\left(-A_{h}^{x}\right)^{n} \tau^{2 n-2} \sum_{\lambda=1}^{n-1}\left(-A_{h}^{x}\right)^{n-\lambda} f^{(2 \lambda-1)}(\tau, x) \\
& -\sum_{m=1}^{j} \delta_{2 m-1}\left(-A_{h}^{x}\right)^{m} \tau^{2 m-2}\left(I+\sum_{n=1}^{l-[m / 2]} a_{2 n}\left(-A_{h}^{x}\right)^{n} \tau^{2 n}\right)^{-1}
\end{aligned}
$$


212 High order of accuracy difference schemes for HDE

$$
\begin{aligned}
& \left\{-\sum_{n=1}^{l-[m / 2]} a_{2 n} \sum_{\lambda=1}^{n}\left(-A_{h}^{x}\right)^{n-\lambda} f^{(2 \lambda-1)}(\tau, x) \tau^{2 n}\right. \\
& \quad-\sum_{n=1}^{l-[m / 2]} a_{2 n-1} \sum_{\lambda=1}^{n}\left(-A_{h}^{x}\right)^{n-\lambda} f^{(2 \lambda-2)}(\tau, x) \tau^{2 n-1} \\
& +\sum_{n=1}^{j-m+[m / 2]} b_{2 n} \sum_{\lambda=1}^{n}\left(-A_{h}^{x}\right)^{n-\lambda} f^{(2 \lambda-1)}(0, x) \tau^{2 n} \\
& \left.\quad+\sum_{n=1}^{j-m+[m / 2]+1} b_{2 n-1} \sum_{\lambda=1}^{n}\left(-A_{h}^{x}\right)^{n-\lambda} f^{(2 \lambda-2)}(0, x) \tau^{2 n-1}\right\} .
\end{aligned}
$$

We have the following.

Theorem 3.4. Let $\tau$ and $|h|$ be sufficiently small numbers. Then the solutions of the difference schemes (3.25) for $l=0$ satisfy the following stability estimates:

$$
\begin{gathered}
\max _{0 \leq k \leq N}\left\|u_{k}^{h}\right\|_{L_{2}\left(\bar{\Omega}_{h}\right)} \\
\leq M_{1}\left[\max _{0 \leq k \leq N-1}\left\|\left(B_{h}^{x}\right)^{-1} f_{k}^{h}\right\|_{L_{2}\left(\bar{\Omega}_{h}\right)}+\left\|\left(B_{h}^{x}\right)^{-1} \psi^{h}\right\|_{L_{2}\left(\bar{\Omega}_{h}\right)}+\left\|\varphi^{h}\right\|_{L_{2}\left(\bar{\Omega}_{h}\right)}\right], \\
\max _{1 \leq k \leq N}\left\|\tau^{-1}\left(u_{k}^{h}-u_{k-1}^{h}\right)\right\|_{L_{2}\left(\bar{\Omega}_{h}\right)} \\
\leq M_{1}\left[\max _{0 \leq k \leq N-1}\left\|f_{k}^{h}\right\|_{L_{2}\left(\bar{\Omega}_{h}\right)}+\left\|\psi^{h}\right\|_{L_{2}\left(\bar{\Omega}_{h}\right)}+\sum_{r=1}^{h}\left\|\varphi_{x_{r}, \bar{m}_{r}}^{h}\right\|_{L_{2}\left(\bar{\Omega}_{h}\right)}\right] \\
\max _{1 \leq k \leq N-1}\left\|\tau^{-2}\left(u_{k+1}^{h}-2 u_{k}^{h}+u_{k-1}^{h}\right)\right\|_{L_{2}\left(b a r \Omega_{h}\right)} \\
\leq M_{1}\left[\left.\max _{1 \leq k \leq N-1}\left\|\tau^{-1}\left(f_{k}^{h}-f_{k-1}^{h}\right)\right\|\right|_{L_{2}\left(\bar{\Omega}_{h}\right)}+\left\|f_{0}^{h}\right\|_{L_{2}\left(\bar{\Omega}_{h}\right)}\right. \\
\left.+\left.\sum_{r=1}^{n}\left\|\psi_{x_{r}, \bar{m}_{r}}^{h}\right\|\right|_{L_{2}\left(\bar{\Omega}_{h}\right)}+\sum_{r=1}^{n}\left\|\left(\varphi_{\bar{x}_{r}}^{h}\right)_{x_{r}, m_{r}}\right\|_{L_{2}\left(\bar{\Omega}_{h}\right)}\right] .
\end{gathered}
$$

Here $M$ does not depend on $\tau, h, \varphi^{h}(x), \psi^{h}(x)$, and $f_{k}^{h}(x), 0 \leq k \leq N-1$.

The proof of Theorem 3.4 is based on the abstract Theorem 3.1, and the symmetry properties of the difference operator $A_{h}^{x}$ is defined by the formula (2.56).

Note that in a similar manner one can construct the difference schemes of a high order of accuracy with respect to one variable for approximate solutions of the boundary value problem (1.17). Abstract Theorem 3.1 permits us to obtain the stability estimates for the solutions of these difference schemes. 


\section{References}

[1] A. Ashyralyev, Difference schemes of a higher order of accuracy for second-order evolution equations, Functional-Differential Equations, Perm. Politekh. Inst., Perm', 1989, pp. 145-150 (Russian).

[2] A. Ashyralyev and H. O. Fattorini, On difference schemes of the high order of accuracy for singular perturbation elliptic equations, Investigation of Theory and Approximation Methods for Differential Equations, Turkmen State University, Ashgabat, 1991, pp. 80-83 (Russian).

[3] - On uniform difference schemes for second-order singular perturbation problems in $\mathrm{Ba}$ nach spaces, SIAM J. Math. Anal. 23 (1992), no. 1, 29-54.

[4] A. Ashyralyev and I. Muradov, On one difference scheme of a second order of accuracy for hyperbolic equations, Proceeding of the IMM of AS of Turkmenistan, no. 3, Ilim, Ashgabat, 1995, pp. 58-63 (Russian).

[5] A. Ashyralyev and P. E. Sobolevskii, A note on the difference schemes for hyperbolic equations, Abstr. Appl. Anal. 6 (2001), no. 2, 63-70.

[6] On the two new approaches for construction of the high order of accuracy difference schemes for the second order differential equations, Funct. Differ. Equ. 10 (2003), no. 3-4, 333-405.

[7] H. O. Fattorini, Second Order Linear Differential Equations in Banach Spaces, North-Holland Mathematics Studies, vol. 108, North-Holland, Amsterdam, 1985.

[8] V. A. Kostin, Analytic semigroups and cosine functions, Dokl. Akad. Nauk SSSR 307 (1989), no. 4, 796-799 (Russian).

[9] S. G. Kreı̆n, Linear Differential Equations in a Banach Space, Nauka, Moscow, 1966.

[10] S. I. Piskarëv, Solution of second-order evolution equations under Kreinn-Fattorini conditions, Differ. Uravn. 21 (1985), no. 9, 1604-1612, 1652 (Russian).

[11] V. Pogorelenko and P. E. Sobolevskii, The "counter-example" to W. Littman counter-example of $L_{p}$-energetical inequality for wave equation, Funct. Differ. Equ. 4 (1997), no. 1-2, p. 165-172 (1998).

[12] P. E. Sobolevskii and L. M. Čebotareva, Approximate solution of the Cauchy problem for an abstract hyperbolic equation by the method of lines, Izv. Vyssh. Uchebn. Zaved. Mat. (1977), no. 5(180), 103-116 (Russian).

Allaberen Ashyralyev: Department of Mathematics, Fatih University, Buyukcekmece, 39400 Istanbul, Turkey

E-mail address: aashyr@fatih.edu.tr

Current address: International Turkmen-Turkish University, 744012 Ashgabat, Turkmenistan

Pavel E. Sobolevskii: Institute of Mathematics, Federal University of Ceará, 60020-181 Fortaleza Ceará, Brazil

Current address: Institute of Mathematics, Hebrew University, Givat Ram, Jerusalem 91904, Israel

E-mail address: pavels@math.huji.ac.il 


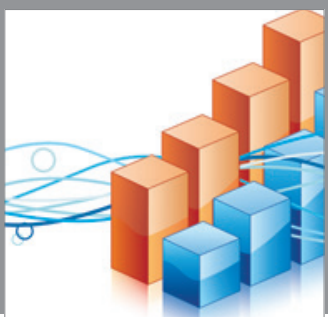

Advances in

Operations Research

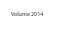

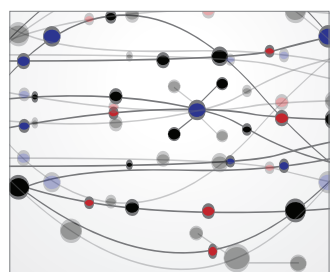

\section{The Scientific} World Journal
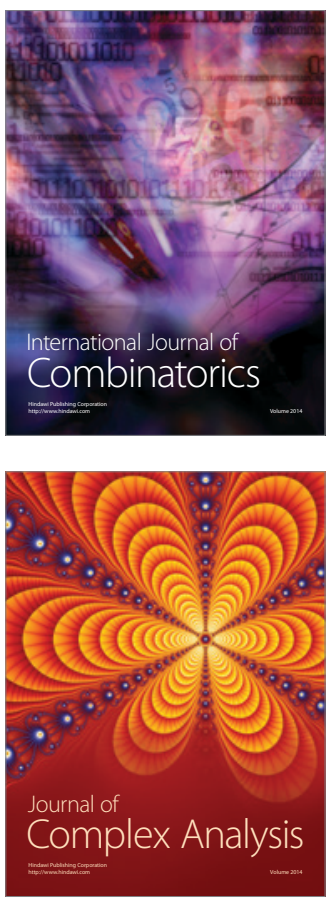

International Journal of

Mathematics and

Mathematical

Sciences
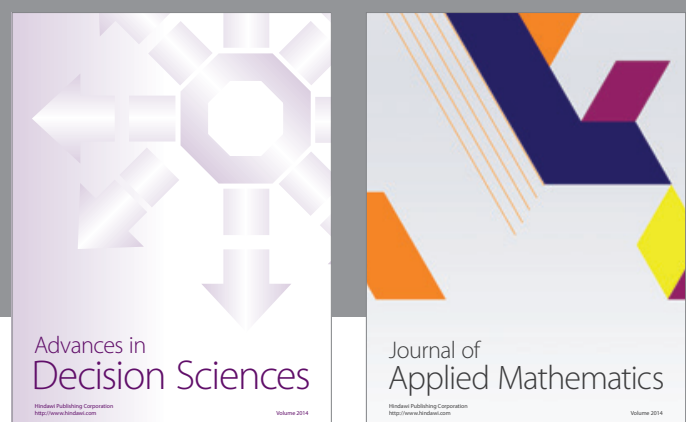

Journal of

Applied Mathematics
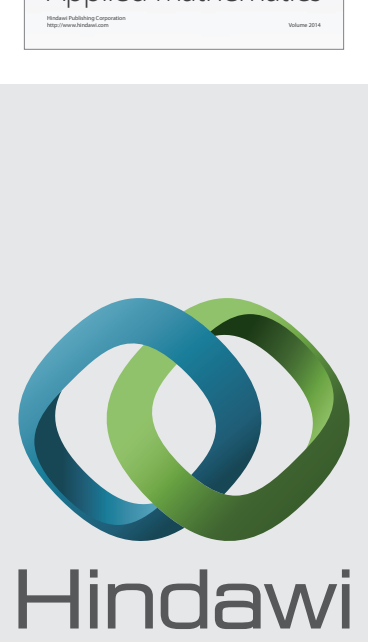

Submit your manuscripts at http://www.hindawi.com
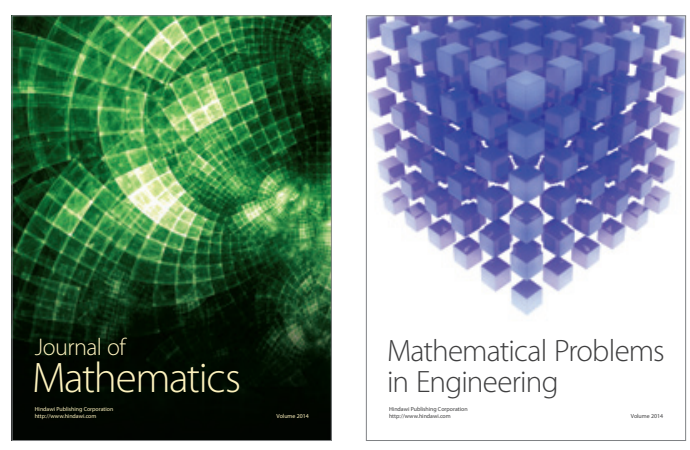

Mathematical Problems in Engineering
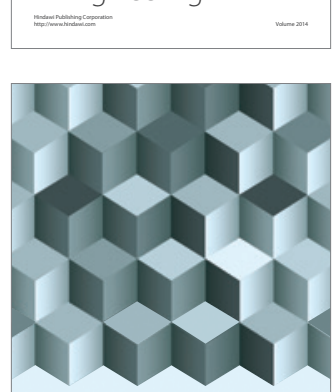

Journal of

Function Spaces
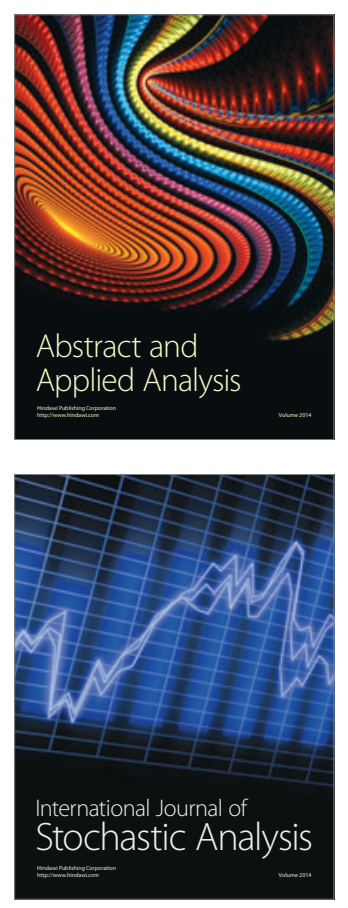

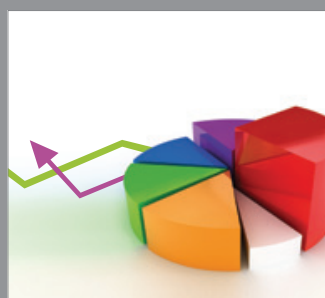

ournal of

Probability and Statistics

Promensencen
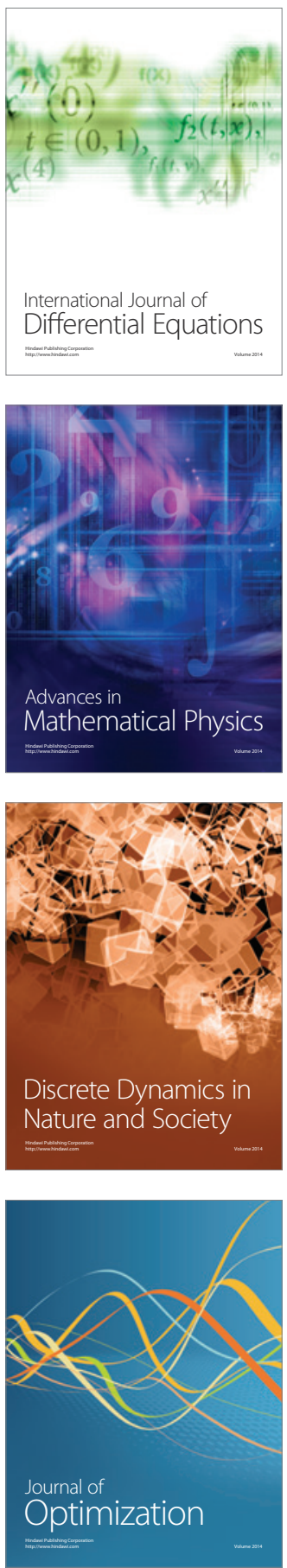